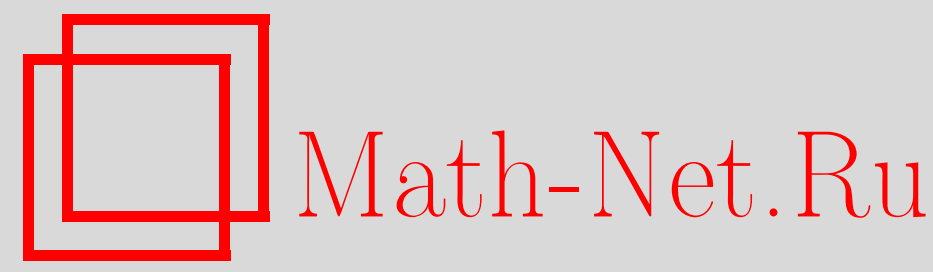

Ю. М. Мовсисян, Алгебры со сверхтождествами многообразия булевых алгебр, Изв. РАН. Сер. матем., 1996, том 60, выпуск 6, 127-168

DOI: https://doi.org/10.4213/im98

Использование Общероссийского математического портала Math-Net.Ru подразумевает, что вы прочитали и согласны с пользовательским соглашением

http://www.mathnet.ru/rus/agreement

Параметры загрузки:

IP: 3.82 .47 .9

26 апреля 2023 г., $17: 55: 14$ 
УДК 512.57

\author{
Ю. М. Мовсисян
}

\title{
Алгебры со сверхтождествами многообразия булевых алгебр
}

\begin{abstract}
В работе дана характеризация алгебр со сверхтождествами многообразия булевых алгебр. Для таких алгебр доказывается структурная теорема. Предварительно характеризуются сверхтождества многообразий решеток, модулярных решеток, дистрибутивных решеток: доказываются теоремы о существовании конечных базисов сверхтождеств.

Библиография: 35 наименований.
\end{abstract}

\section{Введение}

С начала шестидесятых годов в различных разделах алгебры и ее приложениях исследуются следующие два класса абсолютно замкнутых формул из языка второго порядка [1], [2] (применительно к алгебрам) со специализированными кванторами:

$$
\begin{gathered}
\forall X_{1}, \ldots, X_{k} \exists X_{k+1}, \ldots, X_{m} \forall x_{1}, \ldots, x_{n}\left(w_{1}=w_{2}\right), \\
\forall X_{1}, \ldots, X_{m} \forall x_{1}, \ldots, x_{n}\left(w_{1}=w_{2}\right),
\end{gathered}
$$

где $w_{1}, w_{2}$ - термы (слова) от функциональных переменных $X_{1}, \ldots, X_{m}$ и от предметных переменных $x_{1}, \ldots, x_{n}$. Первая формула называется $\forall \exists(\forall)$-тожсдеством, вторая - $\forall(\forall)$-тождеством или сверхтождеством. Выполнимость (истинность) этих формул второго порядка в алгебре $\langle Q ; \Sigma\rangle$ понимается со специализированными кванторами $\left(\forall X_{i}\right)$ и $\left(\exists X_{j}\right)$, означающими: “для каждого значения $X_{i}=A \in \Sigma$ соответствующей арности" и "существует значение $X_{j}=A \in \Sigma$ соответствуюшей арности" . Такая семантика согласована с гомоморфизмами алгебр [3], [4], определяемых как пары $(\varphi, \tilde{\psi})$ отображений с условием

$$
\varphi A\left(x_{1}, \ldots, x_{n}\right)=[\tilde{\psi}(A)]\left(\varphi x_{1}, \ldots, \varphi x_{n}\right) .
$$

Существование фильтрованных и ультрапроизведений в соответствующей категории алгебр $\langle Q ; \Sigma\rangle$ и их гомоморфизмов $(\varphi, \tilde{\psi})$ (в качестве морфизмов) приводит к теореме компактности для абсолютно замкнутых формул второго порядка (без предикатных переменных) со специализированными кванторами $\left(\forall X_{i}\right),\left(\exists X_{j}\right)[4]$. Подобный подход к моделям и алгебраическим системам приводит к теореме компактности для формул второго порядка со специализированными предикатными и функциональными кванторами. Идея специализации кванторов в формулах второго порядка, для получения локальных теорем, восходит к [5]. О языке второго порядка см. также [6], [7]. 
Следуюшие результаты также показывают естественность формул вида (0.1), $(0.2)$ и их моделей (т.е. алгебр с такими формулами).

Хорошо известно, что любое полугрупповое слово с помощью тождества ассоциативности приводится к левонормированной форме расстановки скобок. Если же в слове участвуют различные, например бинарные, операции из алгебры $\langle Q ; \Sigma\rangle$, то любое такое слово с помощью следующего $\forall \exists(\forall)$-тождества ассоциативности:

$$
\forall X, Y \exists X^{\prime}, Y^{\prime} \forall x, y, z\left(X(x, Y(y, z))=X^{\prime}\left(Y^{\prime}(x, y), z\right)\right),
$$

приводится к левонормированной форме расстановки скобок, что легко доказывается индукцией по числу предметных переменных, причем исходное слово может содержать, и лишь одну, неассоциативную (например, квазигрупповую) операцию.

Аналогично, исходя из следующего $\forall \exists(\forall)$-тождества ассоциативности:

$$
\forall X, Y \exists X^{\prime}, Y^{\prime} \forall x, y, z\left(X(Y(x, y), z)=X^{\prime}\left(x, Y^{\prime}(y, z)\right)\right),
$$

любое слово с бинарными операциями приводится к правонормированной форме расстановки скобок. Возникает естественная задача - описание класса алгебр с формулами 2-го порядка (0.3), (0.4). О важности таких постановок задач, например в теории кодирования, см. [8], [9]. Очевидно, вместо $\forall \exists(\forall)$-тождеств ассоциативности здесь можно взять и $\forall(\forall)$-тождества ассоциативности, т.е. сверхтождества ассоциативности (см. также [10], [11]).

Другой результат. Многообразия алгебр характеризуются (обычными) тождествами, однако многообразия многообразий (определяемых как абстрактные классы многообразий алгебр, замкнутых по подмногообразиям, по произведениям многообразий и по редуктам многообразий) уже характеризуются сверхтождествами [12] (см. также [4], где имеется характеризация и квазимногообразий многообразий). Каждая алгебра вкладывается в алгебру функций, тождества которой равносильны сверхтождествам или $\forall \exists(\forall)$-тождествам (П. Кон).

В работе [13] дана характеризация сверхтождеств в многообразии булевых алгебр. Настояшая работа посвяшена характеризации алгебр со сверхтождествами многообразия булевых алгебр (§4). Для таких алгебр доказывается структурная теорема 4.2. Основным здесь является введенная в работе конструкция булевой суммы, сохраняющей сверхтождества булевых алгебр.

В $\S 3$ характеризуются сверхтождества в многообразиях решеток, модулярных решеток и дистрибутивных решеток. Ряд предварительных результатов содержится также и в $\S 1$. Логические и категорные основы теории затрагиваются в $\S 2$.

В качестве приложения доказывается существование такого конечного базиса сверхтождеств многообразия булевых алгебр, для сверхтождеств которого функциональные и предметные ранги не больше 3 . Кроме того, система регулярных тождеств булевых алгебр оказывается кончено базируемой.

\section{§1. Понятие сверхтождества, примеры. Предварительные понятия и результаты}

1.1. Сверхтождество

$$
\forall X_{1}, \ldots, X_{m} \forall x_{1}, \ldots, x_{n}\left(w_{1}=w_{2}\right)
$$


для краткости записывается без кванторной приставки: $w_{1}=w_{2}$. Число $m$ называется функциональным рангом, а число $n-$ предметныц.м рангом сверхтождества.

Будем говорить, что в алгебре $\mathfrak{A}=\langle Q ; \Sigma\rangle$ выполняется сверхтождество

$$
w_{1}=w_{2}
$$

если равенство (1.0) справедливо, когда в нем каждая предметная переменная и каждая функциональная переменная заменяются соответственно любым элементом из $Q$ и любой операцией соответствуюшей арности из $\Sigma$ (предполагается возможность такой замены). Возможность такой замены обеспечивается равенством

$$
T_{\mathfrak{A}}=T_{U(\mathfrak{X})},
$$

где $U(\mathfrak{X})$ - алгебра слов с элементами $w_{1}, w_{2}$, а $T_{\mathfrak{A}}$ - совокупность всех арностей операций из алгебры $\mathfrak{A}$, т.е.

$$
T_{\mathfrak{A}}=\{|A| \mid A \in \Sigma\} \subseteq \mathbb{N},
$$

где $|A|$ - арность операции $A \in \Sigma$, a $\mathbb{N}$ - множество всех натуральных чисел.

$T_{\mathfrak{A}}$ назовем арифметическим типом алгебры $\mathfrak{A}$. T-алгебра - это алгебра с арифметическим типом $T \subseteq \mathbb{N}$. Например, арифметический тип кольца $Q(+, \cdot)$ есть $T=\{2\}$.

Аналогично определяется понятие арифметического типа модели (и алгебраической системы).

Идея алгебры слов приводит к такой $T$-алгебре, множество $n$-арных операций которой $(n \in T)$ имеет сколь угодно большую мощность. Сперва определим арифметический тип для абстрактных множество. Если $U-$ произвольное множество и $\Theta: U \rightarrow \mathbb{N}$ - некоторое отображение этого множества во множество всех натуральных чисел, то образ $\Theta(U) \subseteq \mathbb{N}$ будем называть арифметическим типом $U$. При этом если $\omega \in U$ и $\Theta(\omega)=n$, то $\omega$ назьвается $n$-арныцм, а $n$ - арностью элемента $\omega$ и обозначается $n=|\omega|$. Множество с арифметическим типом $T \subseteq \mathbb{N}$ называется $T$-множеством. Далее стандартным путем определяется понятие алгебры слов с арифметическим типом $T$.

Пусть $T \subseteq \mathbb{N}, \mathfrak{X}$ - произвольное непустое множество, а $U$ - произвольное $T$-множество $(T \neq \varnothing, \mathfrak{X} \cap U=\varnothing)$. Элементы множества $\mathfrak{X}$ называются предметными переменными, а элементы множества $U$ - функииональными переменными. Понятие $T$-слова (или $T$-терма) определяется индуктивно: во-первых, каждое предметное переменное есть $T$-слово, и, во-вторых, если $\omega \in U$ - функциональная переменная с арностью $m$ и $v_{1}, \ldots, v_{m}-T$-слова, то выражение $\omega\left(v_{1}, \ldots, v_{m}\right)$ также есть $T$-слово. Других $T$-слов нет. Равенство двух $T$-слов определяется их графическим совпадением.

Обозначим через $(\mathfrak{X}) U$ совокупность всех $T$-слов. Соответствующая $T$-алгебра $\langle(\mathfrak{X}) U ; U\rangle=U(\mathfrak{X})$ и есть $T$-алгебра слов. $T$-алгебра слов $U(\mathfrak{X})$ называется стандартной, если множество $\mathfrak{X}$ счетно, а множество $U$ для любого $n \in T$ содержит ровно счетное число $n$-арных операций. 
Слово (терм) - это $T$-слово для некоторого $T \subseteq \mathbb{N}$.

Пусть $U(\mathfrak{X})$ - стандартная $T$-алгебра слов, и пусть $\mathfrak{X}=\left\{x_{1}, \ldots, x_{n}, \ldots\right\}$. Естественно выделяется $n$-арный слой $T$-алгебры $U(\mathfrak{X})$, состояший из $n$-арных $T$-слов $(T$-термов):

а) каждый элемент $x_{i}$, где $i=1, \ldots, n$, является $n$-арным $T$-словом;

б) если $w_{1}, \ldots, w_{m}$ являются $n$-арными $T$-словами, то для любого $m$-арного функционального переменного $X \in U, m \in T$, выражение $X\left(w_{1}, \ldots, w_{m}\right)$ также является $n$-арным $T$-словом;

в) $T$-слово называется $n$-арнылм, если оно является $n$-арным согласно а), б).

$n$-арное слово (терм) - это $n$-арное $T$-слово при некотором $T \subseteq \mathbb{N}$.

Класс алгебр называется классом Т-алгебр, если каждая ее алгебра есть $T$-алгебра. Алгебры $\mathfrak{A}$ и $\mathfrak{A}^{\prime}$ называются арифметически однотипными, если $T_{\mathfrak{A}}=T_{\mathfrak{A}^{\prime}}$. Таким образом, группоид и кольцо являются арифметически однотипными алгебрами.

Сверхтождество $w_{1}=w_{2}$ называется $T$-сверхтожсдеством, если $w_{1}, w_{2}$ являются элементами $T$-алгебры слов, т.е. если $w_{1}, w_{2}$ являются $T$-словами.

Систему $T$-сверхтождеств считаем истинной (выполняется) в $T$-алгебре $\mathfrak{A}$, если в $\mathfrak{A}$ выполняется каждое сверхтождество этого семейства; в классе $T$-алгебр выполняется $T$-сверхтождество $w_{1}=w_{2}$, если оно выполняется в любой алгебре этого класса. В частности, можно говорить о сверхтождествах многообразий: сверхтождество $w_{1}=w_{2}$ называется сверхтождеством многообразия $V$, если оно выполняется в каждой алгебре $\mathfrak{A} \in V$. Сверхтождество называется нетривиальнылм, если его функциональный ранг > 1, и тривиальнылм - в противном случае.

$T$-сверхтождество $w_{1}=w_{2}$ называется следствием системы $T$-сверхтождеств $\mathscr{L}$, если всякий раз, когда система $\mathscr{L}$ выполняется в $T$-алгебре, то и сверхтождество $w_{1}=w_{2}$ выполняется в ней, т.е. для любой $T$-алгебры $\mathfrak{A}$

$$
\mathfrak{A} \vDash \mathscr{L} \Longrightarrow \mathfrak{A} \vDash\left(w_{1}=w_{2}\right)
$$

(запись $\mathfrak{A} \vDash \mathscr{L}$ означает выполнимость любого сверхтождества из $\mathscr{L}$ в алгебре $\mathfrak{A}$ ).

ПримеР 1.1. В любой конечной $n$-элементной унарной алгебре $\langle Q ; \Sigma\rangle(|Q|=n)$ выполняется сверхтождество

$$
\underbrace{X(X(\ldots X}_{s}(x) \ldots))=\underbrace{X(X(\ldots X}_{t}(x) \ldots)),
$$

где $s, t \geqslant n-1, s \equiv t\left(\bmod k_{n}\right), k_{n}=\operatorname{HOK}\{1, \ldots, n\}$.

Пример 1.2. В любой решетке $Q(+, \cdot)$ выполняются тривиальные сверхтождества идемпотентности, коммутативности и ассоциативности:

$$
\begin{aligned}
X(x, x) & =x, \\
X(x, y) & =X(y, x), \\
X(x, X(y, z)) & =X(X(x, y), z) .
\end{aligned}
$$


В нетривиальной решетке $Q(+, \cdot)$ не выполняется сверхтождество

$$
X(x, Y(x, y))=x
$$

однако в любой решетке $Q(+, \cdot)$ выполняются следуюшие нетривиальные сверхтождества:

$$
\begin{aligned}
Y(y, x) & =Y(y, X(x, Y(x, y))), \\
Y(X(x, z), y) & =Y(X(x, z), X(y, Y(y, z))) .
\end{aligned}
$$

ПримеР 1.3. В любой решетке $Q(+, \cdot)$ выполняется следующее нетривиальное сверхтождество:

$$
X(z, X(x, Y(x, y)))=X(x, X(z, Y(z, y)))
$$

ПримеР 1.4. Решетка будет модулярной тогда и только тогда, когда в ней выполняется сверхтождество

$$
X(Y(x, X(y, z)), Y(y, z))=Y(X(x, Y(y, z)), X(y, z)) .
$$

Решетка будет дистрибутивной тогда и только тогда, когда в ней выполняются следуюшие нетривиальные сверхтождества дистрибутивности:

$$
\begin{aligned}
& X(x, Y(y, z))=Y(X(x, y), X(x, z)), \\
& X(Y(x, y), z)=Y(X(x, z), X(y, z)) .
\end{aligned}
$$

ПримеР 1.5. В любом конвексоре (или барищентрической алгебре [14], [15]), определяемом как бинарная алгебра с одним сверхтождеством идемпотентности и двумя $\forall \exists(\forall)$-тождествами коммутативности и ассоциативности, выполняются нетривиальные сверхтождества дистрибутивности $\left(\mathrm{d}_{1}\right),\left(\mathrm{d}_{2}\right)^{1}$.

ПримеР 1.6. В любой булевой алгебре $Q\left(+, \cdot{ }^{\prime}\right)$ выполняется следующее нетривиальное сверхтождество:

$$
X\left(Y(x, y)^{\prime}, z\right)^{\prime}=Y\left(X\left(x^{\prime}, z\right)^{\prime}, X\left(y^{\prime}, z\right)^{\prime}\right)
$$

\footnotetext{
${ }^{1}$ Именно с учетом этого факта получена характеризация решеток идеалов конвексоров [4] (задача Л. А. Скорнякова).
} 
1.2. Сверхтождество $w_{1}=w_{2}$ называется однородным (по А.И. Мальцеву), если в словах $w_{1}$ и $w_{2}$ участвуют одни и те же предметные переменные. Очевидно, что любое сверхтождество, выполняюшееся в нетривиальной решетке, является однородным. Поэтому однородным является и любое сверхтождество многообразия решеток.

Следуя Г. Биркгофу [16], систему $\Phi$ подмножеств множества $\{1, \ldots, n\}$ назовем $L$-замкнутой над $\{1, \ldots, n\}$, если она вместе с любым $S \in \Phi$ содержит и произвольное $S^{\prime} \subseteq\{1, \ldots, n\}$ со свойством $S \subseteq S^{\prime}$ (Г. Биркгофом такие системы названы J-замкнутыли).

Рассмотрим $T$-слова при $T=\{2\}$ с не более чем двумя бинарными функциональными переменными $F$ и $G$. Пусть

$$
S=\left\{m_{1}, \ldots, m_{t}\right\} \subseteq\{1, \ldots, n\} .
$$

Обозначим через $\underset{i \in S}{G} x_{i}$ слово от предметных переменных $x_{m_{1}}, \ldots, x_{m_{t}}$ и от бинарного функционального переменного $G$. Аналогично понимается обозначение

$$
\underset{S_{i} \in \Phi}{F}\left\{\underset{k \in S_{i}}{G} x_{k}\right\}
$$

где $\Phi=\left\{S_{1}, \ldots, S_{l}\right\}, S_{1}, \ldots, S_{l} \subseteq\{1, \ldots, n\}$. Если $\Phi=\{S\}$ одноэлементно, то

$$
\underset{S_{i} \in \Phi}{F}\left\{\underset{k \in S_{i}}{G} x_{k}\right\}=\underset{i \in S}{G} x_{i}
$$

если же $S=\{m\}$ одноэлементно, то $\underset{i \in S}{G} x_{i}=x_{m}$.

ОПРЕДЕЛЕНИЕ 1.1. Слово вида

$$
\underset{S_{i} \in \Phi}{F}\left\{\underset{k \in S_{i}}{G} x_{k}\right\}
$$

от бинарных функциональных переменных $F, G$ и от предметных переменных $x_{1}, \ldots, x_{n}$ называется $L$-каноническим и обозначается через $f_{\Phi}\left(F, G, x_{1}, \ldots, x_{n}\right)$, если $\Phi$ является $L$-замкнутой системой над $\{1, \ldots, n\}$ (случай одноэлементного $\Phi$ не исключается).

Два $L$-канонических слова $f_{\Phi}\left(F, G, x_{1}, \ldots, x_{n}\right)$ и $f_{\mathscr{D}}\left(F, G, x_{1}, \ldots, x_{n}\right)$ называются равными, если $\Phi=\mathscr{D}$.

ПРЕДЛОЖЕНИЕ 1.1. Для любого слова $w=\underset{S_{i} \in \mathscr{D}}{F}\left\{\underset{k \in S_{i}}{G} x_{k}\right\}$ существует такое $L$-каноническое слово $\widetilde{w}=f_{\Phi}\left(F, G, x_{1}, \ldots, x_{n}\right)$ с теми эме предметными переменными, что в многообразии решеток выполняется сверхтождество

$$
w=\widetilde{w}
$$

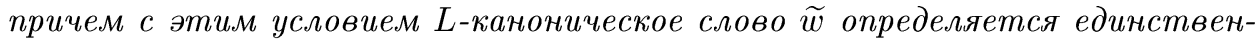
нымм образом и называется L-канонической формой слова $w$; $\widetilde{w}$ получается от слова $w$ после конечного числа переходов, осуществляемых с помощью конечного числа сверхтождеств многообразия решеток, не зависящих от $w$. 
ДокАЗАТЕЛЬСТво. С помошью тривиальных сверхтождеств ассоциативности, коммутативности и идемпотентности многообразия решеток (см. пример 1.2) исключаются повторения предметных переменных $x_{i}$ в подсловах $\underset{k \in S_{i}}{G} x_{k}$. Аналогично исключаются повторения подслов $\underset{k \in S_{i}}{G} x_{k}$ в слове $w$.

Для того чтобы слово

$$
w=\underset{S_{i} \in \mathscr{D}}{F}\left\{\underset{k \in S_{i}}{G} x_{k}\right\}
$$

привести к $L$-каноническому виду, воспользуемся еще сверхтождествами

$$
F(G(y, z), F(x, G(x, y)))=F(G(y, z), x)
$$

и его непосредственным следствием (при $y=z$ )

$$
F(y, F(x, G(x, y)))=F(y, x),
$$

которые выполняются в многообразии всех решеток.

Пусть $S \in \mathscr{D}, S^{\prime} \subseteq\{1, \ldots, n\}$ и $S \subseteq S^{\prime}$. Если в выражении

$$
\underset{S_{i} \in \mathscr{D}}{F}\left\{\underset{k \in S_{i}}{G} x_{k}\right\}
$$

отсутствует $\underset{k \in S^{\prime}}{G} x_{k}$, то это подслово здесь можно получить следующим образом. Допустим $S^{\prime} \backslash S=\{m, k, \ldots\}$, поэтому подслово $\underset{k \in S^{\prime}}{G} x_{k}$ отличается от $\underset{k \in S}{G} x_{k}$ предметными переменными $x_{m}, x_{k}, \ldots$ Поскольку $x_{m}$ участвует в выражении $\underset{S_{i} \in \mathscr{D}}{F}\left\{\underset{k \in S_{i}}{G} x_{k}\right\}$, то, применяя вьшшеуказанные сверхтождества, получим

$$
F\left(G\left(x_{m}, z\right), \underset{k \in S}{G} x_{k}\right)=F\left(G\left(x_{m}, z\right), F\left(\underset{k \in S}{G} x_{k}, G\left(\underset{k \in S}{G} x_{k}, x_{m}\right)\right)\right)
$$

или

$$
F\left(x_{m}, \underset{k \in S}{G} x_{k}\right)=F\left(x_{m}, F\left(\underset{k \in S}{G} x_{k}, G\left(\underset{k \in S}{G} x_{k}, x_{m}\right)\right)\right)
$$

в зависимости от двух возможных расположений $x_{m}$. В обоих случаях возникает новое подслово $G\left(\underset{k \in S}{G} x_{k}, x_{m}\right)$, причем не меняется и прежнее расположение $x_{m}$. Следовательно, после конечного числа аналогичных шагов возникает подслово $\underset{k \in S^{\prime}}{G} x_{k}$.

Первая часть предложения доказана. Докажем единственность. Пусть

$$
w=f_{\Phi_{1}}\left(F, G, x_{1}, \ldots, x_{n}\right)
$$

и

$$
w=f_{\Phi_{2}}\left(F, G, x_{1}, \ldots, x_{n}\right),
$$

где $\Phi_{1} \neq \Phi_{2}$. Допустим, что существует $\left\{i_{1}, \ldots, i_{r}\right\} \subseteq\{1, \ldots, n\}$ такое, что $\left\{i_{1}, \ldots, i_{r}\right\} \in \Phi_{1}$, но $\left\{i_{1}, \ldots, i_{r}\right\} \notin \Phi_{2}$. Поскольку $\Phi_{2}$ является $L$-замкнутым, то справедлива импликация

$$
\left\{l_{1}, \ldots, l_{p}\right\} \subseteq\left\{i_{1}, \ldots, i_{r}\right\} \Longrightarrow\left\{l_{1}, \ldots, l_{p}\right\} \notin \Phi_{2},
$$


т.е. для любого $S \in \Phi_{2}$ существует такое $i \in S$, что $i \notin\left\{i_{1}, \ldots, i_{r}\right\}$. Теперь, рассматривая двухэлементную решетку $B=\{0,1\}$, замечаем, что в этой решетке не выполняется сверхтождество

$$
f_{\Phi_{1}}\left(F, G, x_{1}, \ldots, x_{n}\right)=f_{\Phi_{2}}\left(F, G, x_{1}, \ldots, x_{n}\right) .
$$

Для этого дадим следующие значения всем функциональным и предметным переменным: $F=(+), G=(\cdot), x_{i}=1$, если $i \in\left\{i_{1}, \ldots, i_{r}\right\}, x_{i}=0$, если $i \notin\left\{i_{1}, \ldots, i_{r}\right\}$. Тогда

$$
\underset{k \in\left\{i_{1}, \ldots, i_{r}\right\}}{G} x_{k}=1, \quad f_{\Phi_{1}}\left(F, G, x_{1}, \ldots, x_{n}\right)=1+\cdots=1 .
$$

С другой стороны, согласно вьшесказанному для любого $S \in \Phi_{2}$ сушествует $i \in S$ такое, что $x_{i}=0$. Поэтому

$$
f_{\Phi_{2}}\left(F, G, x_{1}, \ldots, x_{n}\right)=0+\cdots+0=0 .
$$

Итак, в рассматриваемой решетке $B=\{0,1\}$ не выполняется сверхтождество

$$
f_{\Phi_{1}}\left(F, G, x_{1}, \ldots, x_{n}\right)=f_{\Phi_{2}}\left(F, G, x_{1}, \ldots, x_{n}\right) .
$$

Однако по условию

$$
w=f_{\Phi_{1}}\left(F, G, x_{1}, \ldots, x_{n}\right), \quad w=f_{\Phi_{2}}\left(F, G, x_{1}, \ldots, x_{n}\right)
$$

и, следовательно, в многообразии решеток должно выполняться сверхтождество

$$
f_{\Phi_{1}}\left(F, G, x_{1}, \ldots, x_{n}\right)=f_{\Phi_{2}}\left(F, G, x_{1}, \ldots, x_{n}\right) .
$$

Противоречие.

СЛЕДСТВИЕ 1.1. В многообразии всех решеток выполняется сверхтождество

$$
\underset{S_{i} \in \Phi_{1}}{F}\left\{\underset{k \in S_{i}}{G} x_{k}\right\}=\underset{S_{j} \in \Phi_{2}}{F}\left\{\underset{k \in S_{j}}{G} x_{k}\right\}
$$

тогда и только тогда, когда L-канонические формы слов $\underset{S_{i} \in \Phi_{1}}{F}\left\{\underset{k \in S_{i}}{G} x_{k}\right\} u$ $\underset{S_{j} \in \Phi_{2}}{F}\left\{\underset{k \in S_{j}}{G} x_{k}\right\}$ совпадают. 
СЛЕДСТВИЕ 1.2. В многообразии всех решеток любое сверхтождество ви$\partial a$

$$
\underset{S_{i} \in \Phi_{1}}{F}\left\{\underset{k \in S_{i}}{G} x_{k}\right\}=\underset{S_{j} \in \Phi_{2}}{F}\left\{\underset{k \in S_{j}}{G} x_{k}\right\}
$$

является следствием следующих четырех сверхтожсеств (использованных при доказательстве предложсения 1.1):

$$
\begin{aligned}
X(x, x) & =x \\
X(x, y) & =X(y, x), \\
X(x, X(y, z)) & =X(X(x, y), z), \\
X(Y(x, y), X(z, Y(z, x))) & =X(Y(x, y), z) .
\end{aligned}
$$

В качестве приложения $L$-канонических форм докажем справедливость примеpa 1.3. Приведем к $L$-канонической форме левую и правую части рассматриваемого сверхтождества и убедимся, что эти $L$-канонические формы совпадают. Начнем с левой части:

$$
\begin{aligned}
& F(z, F(x, G(x, y)))=F(G(x, y), F(z, x)) \\
& \stackrel{(1.3)}{=} F(G(x, y), F(z, F(x, G(x, z)))) \\
& \quad=F(G(x, z), F(x, F(G(x, y), z))) \\
& \quad \stackrel{(1.2)}{=} F(G(x, z), F(x, F(G(x, y), F(z, G(y, z))))) \\
& \quad \stackrel{(1.3)}{=} F(G(x, z), F(G(x, y), F(z, F(x, F(G(y, z), G(x, G(y, z))))))) \\
& =F(G(G(z, y), x), F(G(x, y), F(G(x, z), F(G(y, z), F(x, z))))) .
\end{aligned}
$$

Теперь приведем к $L$-канонической форме правую часть рассматриваемого сверхтождества:

$$
\begin{aligned}
& F(x, F(z, G(z, y)))=F(G(z, y), F(x, z)) \\
& \stackrel{(1.3)}{=} F(G(z, y), F(x, F(z, G(x, z)))) \\
& \quad=F(G(x, z), F(z, F(G(y, z), x))) \\
& \quad \stackrel{(.2)}{=} F(G(x, z), F(z, F(G(y, z), F(x, G(x, y))))) \\
& \quad \stackrel{(1.3)}{=} F(G(x, z), F(G(x, y), F(z, F(G(y, z), F(x, G(x, G(y, z))))))) \\
& \quad=F(G(G(z, y), x), F(G(x, y), F(G(x, z), F(G(y, z), F(x, z))))) .
\end{aligned}
$$


ПримеР 1.7. В многообразии решеток не выполняется сверхтождество

$$
F(y, F(x, G(x, y)))=G(y, x) .
$$

Действительно, обе части этого сверхтождества, являясь $L$-каноническими формами (словами), не совпадают в смысле равенства $L$-канонических форм. Остается сослаться на следствие 1.1.

TеОРема 1.1. В многообразии дистрибутивных решеток любое сверхтождество функиионального ранга $\leqslant 2$ является следствием следующих четырех сверхтождеств:

$$
\begin{aligned}
X(x, x) & =x, \\
X(x, y) & =X(y, x), \\
X(x, X(y, z)) & =X(X(x, y), z), \\
X(x, Y(y, z)) & =Y(X(x, y), X(x, z)) .
\end{aligned}
$$

ДокАЗАТЕЛЬСТво. Для любого слова $w$ с не более чем двумя бинарными функциональными переменными $F, G$ сушествует такое $L$-каноническое слово $f_{\Phi}\left(F, G, x_{1}, \ldots, x_{n}\right)$ с теми же предметными переменными, что в многообразии дистрибутивных решеток выполняется сверхтождество

$$
w=f_{\Phi}\left(F, G, x_{1}, \ldots, x_{n}\right),
$$

причем с этим условием $L$-каноническое слово определяется единственным образом. Действительно, с помощью сверхтождества дистрибутивности сперва слово $w$ приводится к виду

$$
\underset{S_{i} \in \mathscr{D}}{F}\left\{\underset{k \in S_{i}}{G} x_{k}\right\} \text {. }
$$

Дальше повторяется доказательство предложения 1.1. Остается заметить, что сверхтождество (1.2) является следствием указанных четырех сверхтождеств (идемпотентности, коммутативности, ассоциативности и левой дистрибутивности). Во-первых, очевидно, что сверхтождество правой дистрибутивности

$$
X(Y(y, z), x)=Y(X(y, x), X(z, x))
$$

является следствием сверхтождеств коммутативности и левой дистрибутивности. Далее имеем

$$
\begin{aligned}
X( & x(y, z))=Y(X(x, y), X(x, z)) \\
& =X(Y(x, X(x, z)), Y(y, X(x, z))) \\
& =X(X(Y(x, x), Y(x, z)), X(Y(y, x), Y(y, z))) \\
& =X(X(x, Y(x, z)), X(Y(y, x), Y(y, z))),
\end{aligned}
$$


T.e.

$$
X(x, Y(y, z))=X(X(x, Y(x, z)), X(Y(y, x), Y(y, z))) .
$$

Следовательно,

$$
\begin{aligned}
& X(Y(y, z), X(x, Y(x, y)))=X(X(x, Y(y, z)), Y(x, y)) \\
& \stackrel{(1.4)}{=} X(X(X(x, Y(x, z)), X(Y(y, x), Y(y, z))), Y(x, y)) \\
& \quad=X(X(x, Y(x, z)), X(Y(y, x), Y(y, z))) \\
& \quad=X(x, Y(y, z))=X(Y(y, z), x) .
\end{aligned}
$$

ЗАмЕЧАнИЕ 1.1. Алгебра $Q(+, \cdot)$ с двумя бинарными операциями и со сверхтождествами из следствия 1.2, вообе говоря, не является решеткой. Действительно, в любой нетривиальной решетке $L(+, \cdot)$ выполняются указанные четыре сверхтождества. Рассмотрим прямое произведение $Q=L \times L$ и прямое произведение $\Sigma \times \Sigma$, где $\Sigma=\{+, \cdot\}$. В результате получаем алгебру $Q$ с четырьмя бинарными операциями: $A=(+,+), B=(+, \cdot), C=(\cdot,+), D(\cdot, \cdot)$, причем в этой алгебре $Q(A, B, C, D)$ выполняются указанные четыре сверхтождества. Однако непосредственная проверка показывает, что в алгебре $Q(A, B)$ не выполняются тождества поглошения, поэтому $Q(A, B)$ не является решеткой, хотя в ней также выполняются сверхтождества из следствия 1.2.

ЗАмЕчАниЕ 1.2 . Алгебра $Q(+, \cdot)$ с двумя бинарными операциями и со сверхтождествами из теоремы 1.1, вообще говоря, не является дистрибутивной решеткой.

Пусть $\mathscr{L}$ - некоторый (непустой) набор $T$-сверхтождеств, а $\mathfrak{M}_{\mathscr{L}}^{T}$ - класс всех $T$-алгебр, в которых выполняется каждое сверхтождество из $\mathscr{L}$.

Класс $T$-алгебр $\mathfrak{N}$ называется сверхмногообразием (T-алгебр), если существует система $T$-сверхтождеств $\mathscr{L}$ со свойством

$$
\mathfrak{N}=\mathfrak{M}_{\mathscr{L}}^{T}
$$

При этом $\mathscr{L}$ называется определяющей системой сверхтождеств для $\mathfrak{N}$.

Например, многообразие полугрупп является сверхмногообразием $T$-алгебр, где $T=\{2\}$, а $\mathscr{L}-$ множество из двух сверхтождеств:

$$
\begin{aligned}
X(x, X(y, z)) & =X(X(x, y), z), \\
X(x, y) & =Y(x, y) .
\end{aligned}
$$

Системы $T$-сверхтождеств $\mathscr{L}_{1}, \mathscr{L}_{2}$ называются әквивалентнылми, если

$$
\mathfrak{M}_{\mathscr{L}_{1}}^{T}=\mathfrak{M}_{\mathscr{L}_{2}}^{T}
$$

т.е. каждое $T$-сверхтождество из $\mathscr{L}_{2}$ (из $\left.\mathscr{L}_{1}\right)$ является следствием $\mathscr{L}_{1}$ (соответственно $\mathscr{L}_{2}$ ). 
Будем говорить, что система $T$-сверхтождеств $\mathscr{L}$ имеет конечный базис (или конечно базируема), если она эквивалентна конечной системе $T$-сверхтождеств $\mathscr{L}_{0}$. Каждому классу $T$-алгебр $\mathfrak{M}$ соответствует класс $\Lambda_{\mathfrak{M}}^{T}$ всех таких $T$-сверхтождеств, каждое из которых выполняется во всех алгебрах из $\mathfrak{M}$. Пара отображений

$$
\mathscr{L} \rightarrow \mathfrak{M}_{\mathscr{L}}^{T} \quad \text { и } \quad \mathfrak{M} \rightarrow \Lambda_{\mathfrak{M}}^{T}
$$

образует соответствие Галуа.

Пересечение сверхмногообразий $T$-алгебр - сверхмногообразие $T$-алгебр, а именно:

$$
\bigcap_{i \in I} \mathfrak{M}_{\mathscr{L}_{i}}^{T}=\mathfrak{M}_{\bigcup_{i \in I}^{T}}^{T} \mathscr{L}_{i}
$$

поэтому для любого класса $T$-алгебр $\mathfrak{M}$ сушествует наименьшее (относительно теоретико-множественного включения) сверхмногообразие $T$-алгебр $\mathfrak{M}^{*} \supseteq \mathfrak{M}$, называемое сверхмногообразием алгебр, порожденное $\mathfrak{M}$. Понятно, что $\mathfrak{M}^{*}$ определяется системой $T$-сверхтождеств $\Lambda_{\mathfrak{M}}^{T}$, поэтому характеризация сверхмногообразия $\mathfrak{M}^{*}$ равносильна характеризации всех сверхтождеств класса $T$-алгебр $\mathfrak{M}$.

1.3. Пусть $\mathfrak{A}=\langle Q ; \Sigma\rangle$-алгебра с арифметическим типом $T$, т.е. $T$-алгебра. Понятие $n$-арного $(n \in \mathbb{N})$ полинома $T$-алгебры $\mathfrak{A}$ определяется индуктивно следующим образом:

1) все $n$-арные единичные операции множества $Q$, т.е. операции вида

$$
\delta_{n}^{i}\left(x_{1}, \ldots, x_{n}\right)=x_{i}, \quad i=1, \ldots, n,
$$

являются $n$-арными полиномами алгебры $\mathfrak{A}$;

$2)$ если $m \in T$ и $f_{1}, \ldots, f_{m}$ являются $n$-арньми полиномами алгебры $\mathfrak{A}$, то для любой $m$-арной операции $C \in \Sigma$ следуюшая $n$-арная операция множества $Q$ :

$$
f\left(x_{1}, \ldots, x_{n}\right)=C\left(f_{1}\left(x_{1}, \ldots, x_{n}\right), \ldots, f_{m}\left(x_{1}, \ldots, x_{n}\right)\right),
$$

также является $n$-арным полиномом алгебры $\mathfrak{A}$.

3) $n$-арная операция множества $Q$ называется $n$-арным полиномом алгебры $\mathfrak{A}$, если она является $n$-арным полиномом $\mathfrak{A}$ в силу 1$), 2$ ).

Совокупность всех $n$-арных полиномов $T$-алгебры $\mathfrak{A}=\langle Q ; \Sigma\rangle$ обозначим через $P^{n}(\Sigma)$. Далее, обозначая

$$
P(\Sigma)=P^{1}(\Sigma) \cup P^{2}(\Sigma) \cup \cdots,
$$

получаем алгебру

$$
P(\mathfrak{A})=\langle Q ; P(\Sigma)\rangle,
$$

называемую полиномиальной алгеброй $T$-алгебры $\mathfrak{A}$.

Арифметический тип полиномиальной алгебры всегда равен множеству всех натуральных чисел, поэтому полиномиальные алгебры $P\left(\mathfrak{A}_{1}\right)$ и $P\left(\mathfrak{A}_{2}\right)$ арифметически однотипны для любых $\mathfrak{A}_{1}, \mathfrak{A}_{2}$.

Сверхтождество $w_{1}=w_{2}$ называется полиномиальным сверхтождеством алгебры $\mathfrak{A}$, если оно вьполняется в полиномиальной алгебре $P(\mathfrak{A})$.

Пусть $V$ - многообразие алгебр. Сверхтождество $w_{1}=w_{2}$ назьвается полиномиальныцм сверхтождеством многообразия $V$, если оно является полиномиальныг сверхтождеством для любой алгебры $\mathfrak{A} \in V$. Аналогично определяются полиномиальные сверхтождества класса многообразий $V_{i}, i \in I$. 
ПримеР 1.8. Сверхтождество

$$
X(Y(x, x), Y(x, x))=Y(X(x, x), X(x, x))
$$

является полиномиальным сверхтождеством многообразия групп (полугрупп, луп Муфанг). Здесь имеет место общее сверхтождество вида

$$
\begin{aligned}
& X\left(Y\left(Z_{11}(x), \ldots, Z_{1 m}(x)\right), \ldots, Y\left(Z_{n 1}(x), \ldots, Z_{n m}(x)\right)\right) \\
& =Y\left(X\left(Z_{11}(x), \ldots, Z_{n 1}(x)\right), \ldots, X\left(Z_{1 m}(x), \ldots, Z_{n m}(x)\right)\right)
\end{aligned}
$$

для любых $n, m \in \mathbb{N}$ (при проверке справедливости этого сверхтождества в полиномиальной алгебре лупы Муфанг учитывается теорема Муфанг [17]: в лупе Муфанг подлупа, порожденная любыми двумя элементами, - группа).

ПримеР 1.9. Для любых $n, m \in \mathbb{N}$ сверхтождество

$$
\begin{aligned}
& X\left(Y\left(x_{11}, \ldots, x_{1 m}\right), \ldots, Y\left(x_{n 1}, \ldots, x_{n m}\right)\right) \\
& \quad=Y\left(X\left(x_{11}, \ldots, x_{n 1}\right), \ldots, X\left(x_{1 m}, \ldots, x_{n m}\right)\right)
\end{aligned}
$$

является полиномиальным сверхтождеством многообразия коммутативных групп (полугрупп).

ПримеР 1.10. Сверхтождества

$$
\begin{gathered}
X(x, x)=x \\
X(x, X(y, z))=X(X(x, y), z), \\
X(X(x, y), X(u, v))=X(X(x, u), X(y, v)), \\
X(x, x, x)=x \\
X(x, x, x, x)=x, \\
\ldots \ldots \ldots \ldots \ldots \ldots \ldots
\end{gathered}
$$

являются полиномиальными сверхтождествами многообразия решеток (полурешеток).

Будем говорить, что в алгебре $\mathfrak{A}$ (многообразии $V$ ) полиномиально выполняется сверхтождество $w_{1}=w_{2}$, если $w_{1}=w_{2}$ является полиномиальным сверхтождеством алгебры $\mathfrak{A}$ (многообразия $V)$.

Пусть $\mathscr{L}$ - некоторьй набор сверхтождеств. Через $\mathscr{K}_{\mathscr{L}}$ обозначим класс всех многообразий алгебр, в каждом из которых полиномиально выполняется любое сверхтождество из $\mathscr{L}$. Класс $\mathscr{K}$ многообразий алгебр называется многообразием многообразий, если сушествует система сверхтождеств $\mathscr{L}$ такая, что

$$
\mathscr{K}=\mathscr{K}_{\mathscr{L}} .
$$

В этом случае будем говорить, что многообразие многообразий $\mathscr{K}$ определим системой сверхтождеств $\mathscr{L}$. 
Пересечение многообразий многообразий - многообразие многообразий, а именHO:

$$
\bigcap_{i \in I} \mathscr{K}_{\mathscr{L}_{i}}=\mathscr{K}_{\bigcup_{i \in I}} \mathscr{L}_{i}
$$

поэтому для любого класса $\mathscr{K}$ многообразий алгебр существует наименьшее (относительно теоретико-множественного включения) многообразие многообразий $\mathscr{K}^{*} \supseteq \mathscr{K}$, называемое многообразием многообразий, порожсденное $\mathscr{K}$. Очевидно, $\mathscr{K}^{*}$ определим системой всех полиномиальных сверхтождеств класса многообразий $\mathscr{K}$, поэтому характеризация $\mathscr{K}^{*}$ сводится к характеризации всех полиномиальных сверхтождеств класса многообразий $\mathscr{K}$.

ТЕОРема 1.2 [12]. Абстрактный класс $\mathscr{K}$ многообразий алгебр является многообразием многообразий тогда и только тогда, когда он замкнут по подмногообразиям, по редуктам многообразий и по произведениям многообразий.

ТЕОРема 1.3 [18]. Любое нетривиальное многообразие решеток и многообразие всех полурешеток не имеют конечного базиса полиномиальных сверхтождеств.

TЕОРема 1.4 [19]. Многообразие всех групп (метабелевых групп, моноидов) не имеет конечного базиса полиномиальных сверхтождеств.

ТЕОРема 1.5 [13]. Многообразие булевых алгебр не имеет конечного базиса полиномиальных сверхтождеств.

Сверхтождество $w_{1}=w_{2}$ называется полиномиальным следствием системы сверхтождеств $\mathscr{L}$, если для любой алгебры $\mathfrak{A}$

$$
P(\mathfrak{A}) \vDash \mathscr{L} \Longrightarrow P(\mathfrak{A}) \vDash\left(w_{1}=w_{2}\right),
$$

где $P(\mathfrak{A})$ - полиномиальная алгебра алгебры $\mathfrak{A}$. Здесь естественно возникает и понятие полиномиальной эквивалентности двух систем сверхтождеств.

Следующие утверждения доказываются с использованием канонических форм (см. доказательство предложения 1.1 и теоремы 1.1).

ПРЕДЛОЖЕНИЕ 1.2. Любое полиномиальное сверхтождество с двумя бинарными функииональными переменными многообразия дистрибутивных решеток является полиномиальным следствием следующих сверхтождеств:

$$
\begin{aligned}
X(x, x) & =x, \\
X(x, X(y, z)) & =X(X(x, y), z), \\
X(x, Y(y, z)) & =Y(X(x, y), X(x, z)), \\
X(u, X(x, X(y, v))) & =X(u, X(y, X(x, v))), \\
X(Y(Y(y, x), z), Y(u, v)) & =X(Y(Y(y, x), z), X(Y(Y(u, x), v), Y(u, v))), \\
X(Y(Y(y, x), z), Y(u, x)) & =X(Y(Y(y, x), z), X(Y(Y(y, z), x), Y(u, x))) .
\end{aligned}
$$


ПРЕДЛОЖЕНИЕ 1.3. Любое полиномиальное сверхтождество с унарными функциональными переменными многообразия булевых алгебр является полиномиальным следствием следующих сверхтождеств:

$$
\begin{aligned}
& X(Y(X(X(x))))=X(Y(x)) \\
& X(Y(X(Y(x))))=X(Y(Y(X(x)))) .
\end{aligned}
$$

Об исследованиях сверхтождеств и полиномиальных сверхтождеств в алгебрах и многообразиях алгебр см. также [20]-[29].

\section{§ 2. Категория $T$-алгебр и сверхтождества. Предварительные понятия и результаты}

2.1. Пусть $\mathfrak{A}=\langle Q ; \Sigma\rangle$ и $\mathfrak{A}^{\prime}=\left\langle Q^{\prime} ; \Sigma^{\prime}\right\rangle$ - арифметически однотипные алгебры ${ }^{2}$, или $T_{\mathfrak{A}} \subseteq T_{\mathfrak{A}^{\prime}} ;$ пару $(\varphi, \tilde{\psi})$ отображений $\varphi: Q \rightarrow Q^{\prime}, \tilde{\psi}: \Sigma \rightarrow \Sigma^{\prime}$ назовем гомоморфизмом [3] из алгебры $\mathfrak{A}$ в алгебру $\mathfrak{A}^{\prime}$ и обозначим $(\varphi, \tilde{\psi}): \mathfrak{A} \Longrightarrow \mathfrak{A}^{\prime}$, если отображение $\tilde{\psi}$ сохраняет арность операций и для любой операции $A \in \Sigma,|A|=n$, справедливо равенство

$$
\varphi A\left(x_{1}, \ldots, x_{n}\right)=[\tilde{\psi}(A)]\left(\varphi x_{1}, \ldots, \varphi x_{n}\right)
$$

для любых $x_{1}, \ldots, x_{n} \in Q$.

Помимо полулинейных преобразований $(\varphi, \tilde{\psi})$ линейных пространств, линейных алгебр и модулей, введенные морфизмы охватывают конщепцию слабого гомоморфизма, восходяшего к Марчевскому [30], понятие полиморфизма, введенное Бурбаки, и др.

Для гомоморфизмов $(\varphi, \tilde{\psi})$ стандартным образом вводятся понятия эпиморфизма, мономорфизма, изоморфизма, автоморфизма и эндоморфизма. Гомоморфизм $(\varphi, \tilde{\psi})$ называется эпиморфизмом, если отображения $\varphi, \tilde{\psi}$ сюръективны, и - мономорфизмом, если отображения $\varphi, \tilde{\psi}$ инъективны. Изоморфизм - это одновременно эпи- и мономорфизм. Гомоморфизм (изоморфизм) $(\varphi, \tilde{\psi}) T$-алгебры в себя называется ее эндоморфизмом (автоморфизмом). Множество всех эндоморфизмов (автоморфизмов) $(\varphi, \tilde{\psi})$ одной и той же $T$-алгебры образует полугруппу с единищей (соответственно группу).

В качестве примера отметим законы де Моргана в булевых алгебрах, определяющие автоморфизм $(\varphi, \tilde{\psi})$ булевой алгебры, где $\varphi$-взятие дополнения, а $\tilde{\psi}$ отлично от тождественного.

Арифметически однотипные $T$-алгебры и их гомоморфизмы $(\varphi, \tilde{\psi})$, в качестве морфизмов, образуют категорию, называемую категорией $T$-алгебр, в которой морфизмы между алгебрами меняют не только элементы, но и операции. Следующее утверждение показывает, что $T$-алгебра слов $U(\mathfrak{X})$ - свободный объект этой категории.

\footnotetext{
2 Для простоты в работе рассматриваются алгебры без нульарных операций.
} 
ПРЕДЛОЖЕНИЕ 2.1. Пусть $U(\mathfrak{X})=\langle(\mathfrak{X}) U ; U\rangle$ есть Т-алгебра слов и $\mathfrak{A}=$ $\langle Q ; \Sigma\rangle$ - любая T-алгебра. Если $\tilde{\psi}: U \rightarrow \Sigma-$ произвольное отображение, сохраняющее арность операций, то каждое отображение $\varphi_{0}: \mathfrak{X} \rightarrow Q$ можно продолжить до отображения $\varphi:(\mathfrak{X}) U \rightarrow Q$ maк, что пара $(\varphi, \tilde{\psi})$ есть гомоморфизм из $T$-алгебры слов $U(\mathfrak{X})$ в $T$-алгебру $\mathfrak{A}$ (причем отображение $\varphi$ определяется единственным образом по $\tilde{\psi}$ и $\varphi_{0}$ ).

Действительно, во-первых, определим $\left.\varphi\right|_{\mathfrak{X}}=\varphi_{0}$, и, во-вторых, если $\omega-$ функциональная переменная с арностью $m$ и образы слов $v_{1}, \ldots, v_{m}$ при отображении $\varphi$ уже определены, то образом слова $\omega\left(v_{1}, \ldots, v_{m}\right)$ считаем элемент $\tilde{\psi}(\omega)\left(\varphi v_{1}, \ldots, \varphi v_{m}\right) \in Q$. Следовательно, в $T$-алгебре $\mathfrak{A}=\langle Q ; \Sigma\rangle$ выполняется $T$-сверхтождество $w_{1}=w_{2}$ тогда и только тогда, когда при любом гомоморфизме $(\varphi, \tilde{\psi}): U(\mathfrak{X}) \Longrightarrow \mathfrak{A}$ из $T$-алгебры слов $U(\mathfrak{X})$ в $T$-алгебру $\mathfrak{A}$ справедливо равенство $\varphi\left(w_{1}\right)=\varphi\left(w_{2}\right)$, где $w_{1}, w_{2} \in(\mathfrak{X}) U$.

В категории $T$-алгебр и их гомоморфизмов $(\varphi, \tilde{\psi})$ рассмотрим понятия подалгебры, факторалгебры, прямого и фильтрованного произведения и др.

Алгебра $\mathfrak{A}^{\prime}=\left\langle Q^{\prime} ; \Sigma^{\prime}\right\rangle$ называется подалгеброй алгебры $\mathfrak{A}=\langle Q ; \Sigma\rangle$, если $Q^{\prime} \subseteq Q$ и каждая операция из $\Sigma^{\prime}$ есть ограничение некоторой операции из $\Sigma$ (на подмножестве $\left.Q^{\prime}\right)$. Например, аддитивная полугруппа всех натуральных чисел есть подалгебра кольца цельх чисел, каждая коммутативная группа есть подалгебра некоторого кольца, более того, каждый группоид является подалгеброй некоторого кольца. Каждая полурешетка - подалгебра некоторой дистрибутивной решетки, а каждая дистрибутивная решетка - подалгебра некоторой булевой алгебры и др. Желая указать арифметический тип подалгебр, будем их называть $T$-подалгебрами. Класс $T$-алгебр называется наследственным, если он содержит все $T$-подалгебры любой своей $T$-алгебры.

Промежуточным здесь является следующее понятие подсистемы. Пусть $\mathfrak{A}=$ $\langle Q ; \Sigma\rangle$ - произвольная $T$-алгебра, и пусть $Q^{\prime} \subseteq Q, \Sigma^{\prime} \subseteq \Sigma$, где $Q^{\prime} \neq \varnothing$ и $\Sigma^{\prime} \neq \varnothing$. Пару $\mathfrak{A}^{\prime}=\left\langle Q^{\prime} ; \Sigma^{\prime}\right\rangle$ назовем подсистемой $T$-алгебры $\mathfrak{A}$, если $Q^{\prime}$ замкнуто относительно всех операций из $\Sigma^{\prime}$. Далее, естественно, возникают понятия $T$-подсистемы и подсистемы в подсистеме. Подсистемы вида $\left\langle Q^{\prime} ; \Sigma\right\rangle$ называются злавными.

Каждой подсистеме $\left\langle Q_{1} ; \Sigma_{1}\right\rangle$ алгебры $\langle Q ; \Sigma\rangle$ соответствует ее подалгебра $\left\langle Q_{1} ; \Sigma_{1}^{*}\right\rangle$, если вместо операций из $\Sigma_{1}$ (определенных на множестве $Q$ ) рассматривать их ограничения на подмножестве $Q_{1} \subseteq Q$. Ясно, что каждой $T$-подсистеме соответствует $T$-подалгебра.

Определяя гомоморфизмы $(\varphi, \tilde{\psi})$ между двумя $T$-подсистемами, получим категорию $T$-подсистем.

Класс всех $T$-подсистем (подсистем, главных подсистем) одной и той же $T$-алгебры образует полную и алгебраическую решетку относительно частичного порядка “быть подсистемой” (быть может при добавлении Ø в качестве наименьшего элемента).

Класс всех $T$-подалгебр, а также и класс всех подалгебр одной и той же $T$-алгебры $\mathfrak{A}$ образуют частично упорядоченные множества относительно частичного порядка "быть подалгеброй". Эти частично упорядоченные множества, вообше говоря, не являются решетками. Покажем это на примере пятиэлементной немодулярной решетки $\mathfrak{A}=Q(+, \cdot)$ : 


$$
\begin{gathered}
Q=\{0, a, b, c, 1\}, \\
0<b<a<1, \\
0<c<1, \\
x+y=\sup \{x, y\}, \\
x \cdot y=\inf \{x, y\} .
\end{gathered}
$$

Рассматривая здесь подалгебры $\mathfrak{A}_{1}, \mathfrak{A}_{2}$, соответствуюшие подсистемам $\langle\{a, b, c, 1\} ;\{+\}\rangle$ и $\langle\{a, b, c, 0\} ;\{\cdot\}\rangle$, замечаем, что исходная алгебра $\mathfrak{A}$ обладает лишь тремя такими подалгебрами, которые одновременно являются подалгебрами в $\mathfrak{A}_{1}$ и $\mathfrak{A}_{2}$. Эти три подалгебры соответствуют подсистемам $\langle\{a\} ;\{+, \cdot\}\rangle,\langle\{b\} ;$ $\{+, \cdot\}\rangle,\langle\{c\} ;\{+, \cdot\}\rangle$ и, следовательно, попарно не сравнимы относительно частичного порядка "быть подалгеброй". В силу этого в частично упорядоченном множестве всех подалгебр ( $T$-подалгебр) данной алгебры $\mathfrak{A}$ точная нижняя грань для элементов $\mathfrak{A}_{1}$ и $\mathfrak{A}_{2}$ не сушествует (даже при добавлении $\varnothing$ в качестве наименьшего элемента).

Если $(\varphi, \tilde{\psi}): \mathfrak{A} \Longrightarrow \mathfrak{A}^{\prime}$ - гомоморфизм из $T$-алгебры $\mathfrak{A}$ в $T$-алгебру $\mathfrak{A}^{\prime}$, то пара $\langle\varphi Q ; \tilde{\psi} \Sigma\rangle$ является $T$-подсистемой алгебры $\mathfrak{A}^{\prime}$ и называется гомоморфным.м образом алгебры $\mathfrak{A}$ при гомоморфизме $(\varphi, \tilde{\psi})$. Подалгебра, соответствуюшая подсистеме $\langle\varphi Q ; \tilde{\psi} \Sigma\rangle$, также называется гомоморфным образом алгебры $\mathfrak{A}$ при гомоморфизме $(\varphi, \tilde{\psi})$.

Класс $T$-алгебр называется гомоморфно замкнутым (абстрактным), если он вместе с каждой $T$-алгеброй содержит и каждый ее гомоморфный (изоморфньй) образ при любом гомоморфизме (изоморфизме) $(\varphi, \tilde{\psi})$ (как подалгебра).

Пусть $r$ и $\tilde{t}$ - отношения эквивалентности, определенные соответственно на множествах $Q$ и $\Sigma T$-алгебры $\mathfrak{A}=\langle Q ; \Sigma\rangle$. Пару $q=(r, \tilde{t})$ назовем конгруәнцией $T$-алгебры $\mathfrak{A}$, если, во-первых, $\tilde{t}$ сохраняет арность операций и, во-вторых, отношения $r$ и $\tilde{t}$ согласованы в следуюшем смысле:

$$
x_{1} r x_{1}^{\prime}, \ldots, x_{n} r x_{n}^{\prime}, A \tilde{t} B \rightarrow A\left(x_{1}, \ldots, x_{n}\right) r B\left(x_{1}^{\prime}, \ldots, x_{n}^{\prime}\right),
$$

где $x_{i}, x_{i}^{\prime} \in Q, A, B, \in \Sigma,|A|=|B|=n$.

Если $q=(r, \tilde{t})-$ конгруэнция $T$-алгебры $\mathfrak{A}=\langle Q ; \Sigma\rangle$ и $\tilde{0}$ - тождественное отношение множества $\Sigma$, тогда, очевидно, и $(r, \tilde{0})$ является конгруэнцией $\mathfrak{A}$.

Если $q=(r, \tilde{t})$ - конгруэнция $T$-алгебры $\mathfrak{A}=\langle Q ; \Sigma\rangle$, то каждый элемент $[A] \tilde{t}$ фактормножества $\Sigma / \tilde{t}$ определяет операцию на фактормножестве $Q / r$ следуюшим путем:

$$
[A] \tilde{t}\left(\left[x_{1}\right] r, \ldots,\left[x_{n}\right] r\right)=\left[A\left(x_{1}, \ldots, x_{n}\right)\right] r
$$

где $A \in \Sigma,|A|=n, x_{1}, \ldots, x_{n} \in Q$ и $[\mathfrak{x}] s$ означает класс эквивалентности элемента $\mathfrak{x}$ по отношению эквивалентности $s$.

Корректность определения операции $[A] \tilde{t}$ следует из определения конгруэнции. В результате получаем факторалгебру $T$-алгебры $\mathfrak{A}$ по конгруэнции $q=(r, \tilde{t})$, обозначаемую через $\mathfrak{A} / q$. Конгруэнция $q=(r, \tilde{t}) \quad T$-алгебры $\mathfrak{A}=\langle Q ; \Sigma\rangle$ называется ядерной, если разные элементы фактормножества $\Sigma / \tilde{t}$ определяют разные операции на фактормножестве $Q / r$, т.е.

$$
A \tilde{t} B \Longleftrightarrow A\left(x_{1}, \ldots, x_{n}\right) r B\left(x_{1}, \ldots, x_{n}\right) \quad \forall x_{1}, \ldots, x_{n} \in Q,
$$


где $A, B \in \Sigma,|A|=|B|=n \in T$.

Пусть $\mathfrak{A}=\langle Q ; \Sigma\rangle$ и $\mathfrak{A}^{\prime}=\left\langle Q^{\prime} ; \Sigma^{\prime}\right\rangle-T$-алгебры. С каждым гомоморфизмом $(\varphi, \tilde{\psi}): \mathfrak{A} \Longrightarrow \mathfrak{A}^{\prime}$ связаны две конгруэнции, называемые ядром и слабым ядром гомоморфизма $(\varphi, \tilde{\psi})$. Ядро и слабое ядро гомоморфизма $(\varphi, \tilde{\psi})$ обозначаются соответственно через $\operatorname{Ker}(\varphi, \tilde{\psi})$ и $\operatorname{WKer}(\varphi, \tilde{\psi})$ и определяются как пары

$$
\begin{aligned}
\operatorname{WKer}(\varphi, \tilde{\psi}) & =(\operatorname{Ker} \varphi, \operatorname{Ker} \tilde{\psi}), \\
\operatorname{Ker}(\varphi, \tilde{\psi}) & =(\operatorname{Ker} \varphi, \tilde{t})
\end{aligned}
$$

где

$$
\left.A \tilde{t} B \Longleftrightarrow \tilde{\psi} A\right|_{\varphi(Q)}=\left.\tilde{\psi} B\right|_{\varphi(Q)}, \quad A, B \in \Sigma,
$$

a $\left.C\right|_{S}$ означает ограничение операции $C \in \Sigma^{\prime}$ на подмножестве $S \in Q^{\prime}$. Очевидно, $\operatorname{Ker} \tilde{\psi} \subseteq \tilde{t}$, а если $\varphi-$ сюръекция, то $\tilde{t}=\operatorname{Ker} \tilde{\psi}$.

Для эндоморфизма $(\varphi, \tilde{\varepsilon})$ ненулевого кольца, где $\varphi$-нулевое отображение, а $\tilde{\varepsilon}-$ тождественное отображение, имеем

$$
\operatorname{WKer}(\varphi, \tilde{\varepsilon}) \neq \operatorname{Ker}(\varphi, \tilde{\varepsilon}) .
$$

В общем случае

$$
\operatorname{WKer}(\varphi, \tilde{\psi}) \leqslant \operatorname{Ker}(\varphi, \tilde{\psi})
$$

где частичный порядок “\$” между двумя конгруэнциями определяется естественным путем:

$$
\left(r_{1}, \tilde{t}_{1}\right) \leqslant\left(r_{2}, \tilde{t}_{2}\right) \Longleftrightarrow r_{1} \subseteq r_{2}, \quad \tilde{t}_{1} \subseteq \tilde{t}_{2} .
$$

Класс всех конгруэнций $(r, \tilde{t})$ одной и той же $T$-алгебры образует полную алгебраическую решетку относительно введенного частичного порядка " $\leqslant$ ".

Конгруэнщия $q=(r, \tilde{t}) T$-алгебры $\langle Q ; \Sigma\rangle$ называется вполне инвариантной, если она выдерживает любой эндоморфизм $(\varphi, \tilde{\psi})$ этой алгебры, т.е.

$$
x r y \rightarrow \varphi(x) r \varphi(y)
$$

и

$$
A \tilde{t} B \rightarrow \tilde{\psi}(A) \tilde{t} \tilde{\psi}(B)
$$

где $x, y \in Q, A, B \in \Sigma$.

Перейдем к прямым и фильтрованньм произведениям в рассматриваемой категории. Пусть $\mathfrak{A}_{i}=\left\langle Q_{i} ; \Sigma_{i}\right\rangle, i \in I,-$ арифметически однотипные $T$-алгебры. Образуем декартово произведение $\widehat{Q}=\prod_{i \in I} Q_{i}$ - множество всех функций вида $f: I \rightarrow \bigcup_{i \in I} Q_{i}$, для которых $f(i) \in Q_{i}$ для всех $i \in I$. Далее образуем декартово произведение $\prod_{i \in I} \Sigma_{i}$ и определим подмножество $\widehat{\Sigma} \subseteq \prod_{i \in I} \Sigma_{i}$ как совокупность всевозможных функций $F: I \rightarrow \bigcup_{i \in I} \Sigma_{i}$, удовлетворяющих следующим двум условиям:

$$
\begin{array}{clrl}
F(i) & \in \Sigma_{i} & \text { для всех } & i \in I ; \\
|F(i)| & =|F(j)| & \text { для всех } & i, j \in I,
\end{array}
$$


т.е. при фиксированном $F$ арность операции $F(i) \in \Sigma_{i}$ не зависит от $i \in I$.

Множество $\widehat{\Sigma}$ можно отождествить с совокупностью всевозможных систем $\widehat{A}=$ $\left(A_{i}\right)_{i \in I}$ операций одинаковых арностей, взятых по одному из каждого множества $\Sigma_{i}$. Обозначим $\widehat{\Sigma}=\widetilde{\prod}_{i \in I} \Sigma_{i}$. Если $F \in \widehat{\Sigma}$ и $|F(i)|=n$, то элемент $F$ определяет $n$-арную операцию, определенную на множестве $\widehat{Q}=\prod_{i \in I} Q_{i}$ покомпонентно:

$$
F\left(f_{1}, \ldots, f_{n}\right)(i)=F(i)\left(f_{1}(i), \ldots, f_{n}(i)\right), \quad i \in I,
$$

где $f_{1}, \ldots, f_{n} \in \widehat{Q}$.

В результате возникает $T$-алгебра $\widehat{\mathfrak{A}}=\langle\widehat{Q} ; \widehat{\Sigma}\rangle$, которая и называется прямым произведением $T$-алгебр $\mathfrak{A}_{i}, i \in I$, или их прямьм $T$-произведением и обозначается еще через $\widehat{\mathfrak{A}}=\prod_{i \in I} \mathfrak{A}_{i}$. Естественно определяются подпрямые произведения $T$-алгебр $\mathfrak{A}_{i}, i \in I$, и подпрямая неразложимость в категории $T$-алгебр.

Класс $T$-алгебр называется мультипликативно замкнутым, если он содержит прямое произведение любого семейства $T$-алгебр из заданного класса.

Пусть $\mathfrak{A}_{i}=\left\langle Q_{i} ; \Sigma_{i}\right\rangle, i \in I,-$ арифметически однотипные $T$-алгебры, и пусть $\mathscr{D}-$ фильтр $^{3}$ над множеством $I$. Введем отношение $\equiv \mathscr{D}$, определенное на множестве $\prod_{i \in I} Q_{i}$ и отношение $\sim_{\mathscr{D}}$, определенное на $\widetilde{\prod}_{i \in I} \Sigma_{i}$, полагая

$$
f \equiv \mathscr{D} g \Longleftrightarrow\{\alpha \in I \mid f(\alpha)=g(\alpha)\} \in \mathscr{D},
$$

где $f, g \in \prod_{i \in I} Q_{i}$, и

$$
F \sim_{\mathscr{D}} G \Longleftrightarrow\{\alpha \in I \mid F(\alpha)=G(\alpha)\} \in \mathscr{D},
$$

где $F, G \in \widetilde{\prod}_{i \in I} \Sigma_{i},|F|=|G|$.

Согласно определению фильтра отношения $\equiv_{\mathscr{D}}$ и $\sim_{\mathscr{D}}$ будут отношениями эквивалентности. Далее, легко доказывается, что пара эквивалентностей $\left(\equiv \mathscr{D}, \sim_{\mathscr{D}}\right)$ будет и конгруэнцией прямого произведения $T$-алгебр $\mathfrak{A}_{i}=\left\langle Q_{i} ; \Sigma_{i}\right\rangle, i \in I$. Соответствуюшая факторалгебра обозначается через $\prod_{i \in I} \mathfrak{A}_{i} / \mathscr{D}$ и называется $\phi и л ь т-$ рованным (по фильтру $\mathscr{D}$ ) произведением $T$-алгебр $\mathfrak{A}_{i}=\left\langle Q_{i} ; \Sigma_{i}\right\rangle, i \in I$. Фильтрованное по ультрафильтру произведение $T$-алгебр называется ультрапроизведением T-алгебр.

Конгруэнция $\left(\equiv \mathscr{D}, \sim_{\mathscr{D}}\right)$ будет ядерной. Действительно, для любых $f_{1}, \ldots, f_{n} \in$ $\prod_{i \in I} Q_{i}$ имеем

$$
\begin{aligned}
F \sim_{\mathscr{D}} G & \Longleftrightarrow\{\alpha \in I \mid F(\alpha)=G(\alpha)\} \in \mathscr{D} \\
& \Longleftrightarrow\left\{\alpha \in I \mid F(\alpha)\left(f_{1}(\alpha), \ldots, f_{n}(\alpha)\right)=G(\alpha)\left(f_{1}(\alpha), \ldots, f_{n}(\alpha)\right)\right\} \in \mathscr{D} \\
& \Longleftrightarrow\left\{\alpha \in I \mid F\left(f_{1}, \ldots, f_{n}\right)(\alpha)=G\left(f_{1}, \ldots, f_{n}\right)(\alpha)\right\} \in \mathscr{D}
\end{aligned}
$$

\footnotetext{
${ }^{3}$ фильтром над непустым множеством $I$ называется любая непустая совокупность $\mathscr{D}$ подмножеств множества $I$, удовлетворяющая требованиям:

а) пересечение любых двух подмножеств из $\mathscr{D}$ принадлежит $\mathscr{D}$;

б) все надмножества любого подмножества, принадлежащего $\mathscr{D}$, также принадлежат $\mathscr{D}$;

c) пустое множество $\varnothing$ не принадлежит $\mathscr{D}$.

Максимальный фильтр над $I$, т.е. фильтр над $I$, не лежащий ни в каком другом фильтре над $I$, обычно называется ультрафильтром над $I$.
} 


$$
\Longleftrightarrow F\left(f_{1}, \ldots, f_{n}\right) \equiv_{\mathscr{D}} G\left(f_{1}, \ldots, f_{n}\right) .
$$

Совокупность $T$-сверхтождеств назьвается выполнимой, если сушествует (неодноэлементная) $T$-алгебра, в которой истинно каждое $T$-сверхтождество указанной совокупности. Теорема компактности языка 1-й ступени с помощью ультрапроизведений $T$-алгебр распространяется на сверхтождества [4].

ТЕОРема 2.1. Если выполнима каждая конечная часть бесконечной совокупности Т-сверхтождеств, то выполнима и вся совокупность Т-сверхтождеств.

Сверхмногообразия $T$-алгебр естественно характеризуются введенньми категорными понятиями [3], [4].

Теорема 2.2. Класс Т-алгебр будет сверхмногообразием тогда и только тогда, когда он наследствен, гомоморфно и мультипликативно замкнут.

Прямое $T$-произведение двух ненулевых колец (решеток), как произведение $T$-алгебр, где $T=\{2\}$, являясь алгеброй с четырьмя операциями, не является кольцом (решеткой). Однако из следуюшего предложения 2.2 (ср. [31]) вытекает, что ультрапроизведение конечного числа конечных колец (решеток), как ультрапроизведение $T$-алгебр, является кольцом (решеткой).

ПРЕДЛОЖЕНИЕ 2.2. Пусть $\mathfrak{A}_{0}, \ldots, \mathfrak{A}_{n-1}$ - конечные Т-алгебры с конечныцми множествами операций, и пусть $\mathfrak{A}_{i}, i \in I$, - непустое семейство T-алгебр, каждая из которых есть одна из алгебр $\mathfrak{A}_{0}, \ldots, \mathfrak{A}_{n-1}$. Если $\mathscr{D}$ - ультрафильтр над $I$, то существует число $j, 0 \leqslant j<n$, такое, что имеем изоморфность

$$
\prod_{i \in I} \mathfrak{A}_{i} / \mathscr{D} \simeq \mathfrak{A}_{j} .
$$

ДокАЗАТЕЛЬСТво. Определим множества

$$
I_{j}=\left\{i \in I \mid \mathfrak{A}_{i}=\mathfrak{A}_{j}\right\}, \quad 0 \leqslant j<n,
$$

и заметим равенство $I=I_{0} \cup I_{1} \cup \cdots \cup I_{n-1}$. Поскольку фильтр $\mathscr{D}$ простой и $I \in \mathscr{D}$, то существует $j \in\{0, \ldots, n-1\}$ такой, что $I_{j} \in \mathscr{D}$. В силу ядерности конгруэнций $\left(\equiv_{\mathscr{D}}, \sim_{\mathscr{D}}\right)$ имеем

$$
\prod_{i \in I} \mathfrak{A}_{i} / \mathscr{D} \simeq \prod_{i \in I_{j}} \mathfrak{A}_{i} / \mathscr{E}
$$

где $\mathscr{E}=\left\{X \cap I_{j} \mid X \in \mathscr{D}\right\}$ - ультрафильтр над $I_{j}$.

Для $a \in Q_{j}$ определим $f_{a} \in \prod_{i \in I_{j}} Q_{i}$ со свойством $f_{a}(i)=a$ для всех $i \in I_{j}$. Для $X \in \Sigma_{j}$ определим $F_{X} \in \widetilde{\prod}_{i \in I_{j}} \Sigma_{i}$ со свойством $f_{X}(i)=X$ для всех $i \in I_{j}$. Если $a \neq b$ и $a, b \in Q_{j}$, то

$$
\left\{\alpha \in I_{j} \mid f_{a}(\alpha)=f_{b}(\alpha)\right\}=\varnothing \notin \mathscr{E} .
$$

Следовательно, $f_{a} \not \equiv \mathscr{E} f_{b}$ и отображение $\alpha: a \rightarrow\left[f_{a}\right]_{\mathscr{E}}$, где $\left[f_{a}\right]_{\mathscr{E}}-$ класс эквивалентности по отношению $\equiv \mathscr{E}$, будет инъекцией. Аналогично показывается, что 
отображение $\tilde{\alpha}: X \rightarrow\left[F_{X}\right]_{\mathscr{E}}$, где $\left[F_{X}\right]_{\mathscr{E}}-$ класс эквивалентности по отношению $\sim_{\mathscr{E}}$, будет инъекций. Далее, исходя из равенства $f_{X\left(a_{1}, \ldots, a_{n}\right)}=F_{X}\left(f_{a_{1}}, \ldots, f_{a_{n}}\right)$, проверяется условие гомоморфности для пары $(\alpha, \tilde{\alpha}): \mathfrak{A}_{j} \Longrightarrow \prod_{i \in I_{j}} \mathfrak{A}_{i} / \mathscr{E}:$

$$
\begin{aligned}
& \left.\alpha X\left(a_{1}, \ldots, a_{n}\right)=\left[f_{X\left(a_{1}, \ldots, a_{n}\right)}\right)\right]_{\mathscr{E}}=\left[F_{X}\left(f_{a_{1}}, \ldots, f_{a_{n}}\right)\right]_{\mathscr{E}} \\
& =\left[F_{X}\right]_{\mathscr{E}}\left(\left[f_{a_{1}}\right]_{\mathscr{E}}, \ldots,\left[f_{a_{n}}\right]_{\mathscr{E}}\right)=[\tilde{\alpha}(X)]\left(\alpha a_{1}, \ldots, \alpha a_{n}\right) .
\end{aligned}
$$

Остается проверить сюръективность $\alpha, \tilde{\alpha}$.

2.2. Любое $T$-сверхтождество является и $T^{\prime}$-сверхтождеством для любого множества натуральных чисел $T^{\prime} \supseteq T$. Очевидно, что если $w_{1}=w_{2}$ является следствием $\mathscr{L}$ в смысле $T$-сверхтождеств $(\S 1)$, то $w_{1}=w_{2}$ будет следствием $\mathscr{L}$ и в смысле $T^{\prime}$-сверхтождеств, где $T^{\prime} \supseteq T$.

Перейдем к понятию формального следствия системы $T$-сверхтождеств $\mathscr{L}$.

(i) Любое $T$-сверхтождество вида $w=w$ и любое $T$-сверхтождество из $\mathscr{L}$ являются формальными следствиями $\mathscr{L}$.

(ii) Если $T$-сверхтождество $w_{1}=w_{2}$ является формальным следствием $\mathscr{L}$, то и сверхтождество $w_{2}=w_{1}$ является формальным следствием $\mathscr{L}$.

(iii) Если $T$-сверхтождества $w_{1}=w_{2}$ и $w_{2}=w_{3}$ являются формальными следствиями $\mathscr{L}$, то и $T$-сверхтождество $w_{1}=w_{3}$ является формальным следствием $\mathscr{L}$.

(iv) Если $T$-сверхтождество $u_{i}=v_{i}$ является формальньм следствием $\mathscr{L}$ для $i=1, \ldots, n$, то для любого $n$-арного функционального переменного $Y$ (где $n \in T$ ) $T$-сверхтождество

$$
Y\left(u_{1}, \ldots, u_{n}\right)=Y\left(v_{1}, \ldots, v_{n}\right)
$$

также является формальным следствием $\mathscr{L}$.

(v) Если $T$-сверхтождество $w_{1}=w_{2}$ является формальным следствием $\mathscr{L}$, то и $T$-сверхтождество $w_{1}^{\prime}=w_{2}^{\prime}$ является формальным следствием $\mathscr{L}$, причем $w_{1}^{\prime}$ и $w_{2}^{\prime}$ получаются соответственно из $w_{1}$ и $w_{2}$ заменой всех вхождений произвольного функционального переменного $X$ (предметного переменного $x$ ) на любое функциональное переменное той же арности (соответственно на любое $T$-слово $\omega$ ).

$T$-сверхтождество $w_{1}=w_{2}$ называется формальныц следствием системы T-сверхтождеств $\mathscr{L}$, если оно является формальным следствием $\mathscr{L}$ согласно (i) $-(\mathrm{v})$.

ТЕОРЕМА 2.3 (О ПОЛНОТЕ ДЛЯ СВЕРХТОЖДЕСТВ). T-сверхтождество $w_{1}=w_{2}$ является следствием системы $T$-сверхтождеств $\mathscr{L}$ тогда и только тогда, когда оно является формальным следствием $\mathscr{L}$ [3], [4].

Пусть $T=\mathbb{N}$ - множество всех натуральных чисел.

Перейдем к понятию формального полиномиального следствия системы $\mathbb{N}$-сверхтождеств $\mathscr{L}$.

(i') Совпадает с (i) при $T=\mathbb{N}$.

(ii') Совпадает с (ii) при $T=\mathbb{N}$.

(iii') Совпадает с (iii) при $T=\mathbb{N}$.

(iv') Совпадает с (iv) при $T=\mathbb{N}$.

$\left(\mathrm{v}^{\prime}\right)$ Совпадает с (v) при $T=\mathbb{N}$. 
(vi') Если $\mathbb{N}$-сверхтождество $w_{1}=w_{2}$ является формальным полиномиальным следствием $\mathscr{L}$, то и $\mathbb{N}$-сверхтождество $w_{1}^{\prime \prime}=w_{2}^{\prime \prime}$ является формальным полиномиальным следствием $\mathscr{L}$, причем $w_{1}^{\prime \prime}$ и $w_{2}^{\prime \prime}$ получаются соответственно из $w_{1}$ и $w_{2}$ заменой всех вхождений произвольного функционального переменного $X$ на произвольный терм той же арности.

$\mathbb{N}$-сверхтождество $w_{1}=w_{2}$ называется формальным полиномиальным следствием системы $\mathbb{N}$-сверхтождеств $\mathscr{L}$, если оно является формальным полиномиальным следствием $\mathscr{L}$ согласно $\left(\mathrm{i}^{\prime}\right)-\left(\mathrm{vi}^{\prime}\right)$.

ТЕОРЕМА 2.4 (О ПОЛНОТЕ ДЛЯ ПОЛИНОМИАЛЬНЫХ СВЕРХТОЖДЕСТВ). $\mathbb{N}$-сверхтождество $w_{1}=w_{2}$ является полиномиальным следствием системьи $\mathbb{N}$-сверхтохсдеств $\mathscr{L}$ тогда и только тогда, когда оно является формальным полиномиальным следствием $\mathscr{L}$.

Доказательство теоремы 2.4 - повторение доказательства теоремы 2.3 с использованием понятия полиномиально инвариантной конгруэнщии вместо понятия вполне инвариантной конгруэнщии.

Конгруэнщия $r=(r, \tilde{0}) \quad T$-алгебры $\mathfrak{A}=\langle Q ; \Sigma\rangle$ называется полиномиально инвариантной, если она выдерживает любой гомоморфизм $(\alpha, \tilde{\beta}): \mathfrak{A} \Longrightarrow P(\mathfrak{A})$, т.e.

$$
x r y \rightarrow \alpha(x) r \alpha(y)
$$

где $x, y \in Q$, а $P(\mathfrak{A})$ - полиномиальная алгебра алгебры $\mathfrak{A}$.

\section{§3. Сверхтождества решеток}

ТЕОрема 3.1. Любое сверхтождество многообразия дистрибутивных реиеток является следствием следуюших четырех сверхтождеств:

$$
\begin{aligned}
X(x, x) & =x, \\
X(x, y) & =X(y, x), \\
X(x, X(y, z)) & =X(X(x, y), z), \\
X(x, Y(y, z)) & =Y(X(x, y), X(x, z)) .
\end{aligned}
$$

Требуется доказать, что если в $T$-алгебре $\mathfrak{A}$, где $T=\{2\}$, выполняются сверхтождества (3.1)-(3.4), то в ней выполняется и любое сверхтождество многообразия дистрибутивных решеток.

ОПРЕДЕЛЕНИЕ 3.1. Бинарную алгебру $\mathfrak{A}=\langle Q ; \Sigma\rangle$ назовем

а) квазирешеткой, если она удовлетворяет сверхтождествам (3.1)-(3.4);

б) а-квазирешеткой, если она квазирешетка и сушествует натуральное число $n \geqslant 2$ такое, что в алгебре $\mathfrak{A}=\langle Q ; \Sigma\rangle$ выполняется следующая формула:

$$
\exists X_{1}, \ldots, X_{n} \forall x, y\left(X_{1}\left(x, \ldots X_{n-1}\left(x, X_{n}(x, y)\right) \ldots\right)=x\right)
$$

(значения функциональных переменных $X_{1}, \ldots, X_{n}$ предполагаются из $\Sigma$ ).

Если множество $\Sigma$ конечно и $|\Sigma|=n$, то такие квазирешетки под названием дистрибутивных $n$-квазирешеток изучаются в [32]. В случае $n=2$ см. также [33], [34]. 
ПримеР 3.1. Прямое $T$-произведение решеток, где $T=\{2\}$, является $a$-квазирешеткой.

В категории $T$-алгебр $a$-квазирешетки оказываются подалгебрами прямых произведений дистрибутивных решеток. Начнем с характеризаций подпрямо-неразложимых квазирешеток в категории $T$-алгебр.

ЛЕмма 3.1. Если квазирешетка $\mathfrak{A}=\langle Q ; \Sigma\rangle$ подпрямо-неразложима в категории T-алгебр, где $T=\{2\}$, то мощность $|\Sigma|$ не больще 2.

ДокАЗАТЕЛЬСтво. В случае $|\Sigma| \geqslant 3$ квазирешетка $\mathfrak{A}=\langle Q ; \Sigma\rangle$ оказывается подпрямо-разложимой. Действительно, пусть $A_{1}, A_{2}, A_{3}$ - попарно различные бинарные операции из $\Sigma$, и пусть

$$
f_{i, j}(x, y)=A_{i}\left(x, A_{j}(x, y)\right)
$$

Определим отношения $\Theta_{i, j}$ на множества $Q$ следуюшим образом:

$$
x \Theta_{i, j} y \Longleftrightarrow f_{i, j}(x, y)=x, f_{i, j}(y, x)=y,
$$

где $i, j=1,2,3$ и $i<j$. Реффлексивность и симметричность отношения $\Theta_{i, j}$ очевидны, а транзитивность вытекает из сверхтождества

$$
X(x, Y(x, X(y, Y(y, z))))=X(x, Y(x, X(z, Y(z, y))))
$$

которое является следствием сверхтождеств (3.1)-(3.4) и, следовательно, выполняется в любой квазирешетке. Действительно, сверхтождество (1.2) является следствием сверхтождеств (3.1)-(3.4) (см. доказательство теоремы 1.1). Записывая сверхтождество (1.2) в виде (сверхтождества (3.1)-(3.3) применяются без оговорки)

$$
X(x, X(Y(x, y), Y(y, z)))=X(x, Y(y, z))
$$

и заменяя $y \rightarrow Y(x, y)$, получим сверхтождество

$$
X(x, X(Y(x, y), Y(Y(x, y), z)))=X(x, Y(Y(x, y), z)) .
$$

Следовательно, в любой квазирешетке выполняется и сверхтождество

$$
X(x, X(Y(x, z), Y(Y(x, y), z)))=X(x, Y(Y(x, y), z)) .
$$

Из последних двух сверхтождеств с использованием сверхтождества (3.4) получаем сверхтождество (3.5).

В дальнейшем нам понадобится следующее сверхтождество:

$$
X(Y(x, y), z)=X(X(Y(x, y), z), Y(Y(x, y), z))
$$

являюшееся очевидным следствием сверхтождества (1.3). 
Докажем, что $\Theta_{i, j}=\left(\Theta_{i, j}, \tilde{0}\right)$ является конгруэнщией квазирешетки $\mathfrak{A}=\langle Q ; \Sigma\rangle$, т.е. если $x \Theta_{i, j} y$ и $u \Theta_{i, j} v$, то $A(x, u) \Theta_{i, j} A(y, v)$ для любой операции $A \in \Sigma$. Достаточно показать, что $A(x, u) \Theta_{i, j} A(y, u)$, где $x \Theta_{i, j} y$ и $x, y, u \in Q$. Воспользуемся сверхтождеством

$$
X(Y(X(x, y), z), Y(y, z))=Y(X(x, y), z),
$$

также являющимся следствием сверхтождеств (3.1)-(3.4):

$$
\begin{aligned}
& X(Y(X(x, y), z), Y(y, z))=X(X(Y(x, z), Y(y, z)), Y(y, z)) \\
& =X(Y(x, z), X(Y(y, z), Y(y, z)))=X(Y(x, z), Y(y, z))=Y(X(x, y), z) .
\end{aligned}
$$

Пусть $x \Theta_{i, j} y$, т.е. $A_{i}\left(x, A_{j}(x, y)\right)=x$ и $A_{i}\left(y, A_{j}(y, x)\right)=y$, где $A_{i}, A_{j} \in \Sigma$. Тогда для любой операции $A \in \Sigma$ имеем

$$
\begin{aligned}
A(x, u) & =A\left(A_{i}\left(x, A_{j}(x, y)\right), u\right) \\
& \stackrel{(3.8)}{=} A_{i}\left(A\left(A_{i}\left(x, A_{j}(x, y)\right), u\right), A\left(A_{j}(x, y), u\right)\right) \\
& \stackrel{(3.4)}{=} A_{i}\left(A(x, u), A_{j}(A(x, u), A(y, u))\right) .
\end{aligned}
$$

Аналогично получаем равенство

$$
A(y, u)=A_{i}\left(A(y, u), A_{j}(A(y, u), A(x, u))\right) .
$$

Заметим, что доказательство свойства конгруэнции отношения $\Theta_{i, j}$ можно осуществить и более слабыми средствами, а именно вместо сверхтождеств $(3.8),(3.4)$ (или его двойственного сверхтождества правой дистрибутивности) можно воспользоваться сверхтождествами (3.6), (3.7), а также сверхтождеством

$$
X(X(Y(X(x, y), z), Y(x, z)), Y(y, z))=Y(X(x, y), z),
$$

являюшимся следствием сверхтождества (3.8). Действительно, если $x \Theta_{i, j} y$, то для любого $u \in Q$ и $A \in \Sigma$, обозначая $a=A(x, u), b=A(y, u), c=A\left(A_{j}(x, y), u\right)$, $t=A_{i}\left(x, A_{j}(x, y)\right), s=A(t, u)$, получим

$$
\begin{aligned}
A(x, u) & =A\left(A_{i}\left(x, A_{j}(x, y)\right), u\right) \\
& \stackrel{(3.9)}{=} A_{i}\left(A_{i}\left(A\left(A_{i}\left(x, A_{j}(x, y)\right), u\right), A(x, u)\right), A\left(A_{j}(x, y), u\right)\right) \\
& \stackrel{(3.1)}{=} A_{i}\left(A_{i}(s, a), A_{i}\left(A\left(A_{j}(x, y), u\right), c\right)\right) \\
& \stackrel{(3.9)}{=} A_{i}\left(A_{i}(s, a), A_{i}\left(A_{j}\left(A_{j}\left(A\left(A_{j}(x, y), u\right), A(x, u)\right), A(y, u)\right), c\right)\right) \\
& =A_{i}\left(A_{i}(s, a), A_{i}\left(A_{j}\left(A_{j}(c, a), b\right), c\right)\right) \\
& =A_{i}\left(s, A_{i}\left(A_{i}\left(a, A_{j}\left(A_{j}(a, b), c\right)\right), c\right)\right) \\
& \stackrel{(3.6)}{=} A_{i}\left(s, A_{i}\left(A_{i}\left(a, A_{i}\left(A_{j}(a, b), A_{j}\left(A_{j}(a, b), c\right)\right)\right), c\right)\right) \\
& =A_{i}\left(s, A_{i}\left(a, A_{i}\left(A_{i}\left(A_{j}(a, b), c\right), A_{j}\left(A_{j}(a, b), c\right)\right)\right)\right) \\
& \stackrel{(3.7)}{=} A_{i}\left(s, A_{i}\left(a, A_{i}\left(A_{j}(a, b), c\right)\right)\right)=A_{i}\left(A_{i}\left(A_{i}(s, a), c\right), A_{j}(a, b)\right) \\
& \stackrel{(3.9)}{=} A_{i}\left(s, A_{j}(a, b)\right)=A_{i}\left(A(x, u), A_{j}(A(x, u), A(y, u))\right) .
\end{aligned}
$$


Аналогично получается второе равенство согласованности.

Рассмотрим факторалгебры $\mathfrak{A} / \Theta_{i, j}$ по конгруэнциям $\Theta_{1,2}, \Theta_{1,3}$ и $\Theta_{2,3}$. Отображения

$$
\varphi_{0}: x \rightarrow\left([x]_{1,2},[x]_{1,3},[x]_{2,3}\right), \quad \tilde{\psi}_{0}: A \rightarrow\left([A]_{1,2},[A]_{1,3},[A]_{2,3}\right)
$$

определяют мономорфизм квазирешетки $\mathfrak{A}$ в прямое произведение $\mathfrak{A} / \Theta_{1,2} \times$ $\mathfrak{A} / \Theta_{1,3} \times \mathfrak{A} / \Theta_{2,3}$. Гомоморфность этой пары очевидна, а инъективности отображения $\varphi_{0}$, следовательно, и отображения $\tilde{\psi}_{0}$ вытекают из следующей импликации: если

$$
f_{1,2}(x, y)=f_{1,3}(x, y)=f_{2,3}(x, y)=x
$$

то

$$
x=A_{1}\left(A_{2}(x, y), A_{3}(x, y)\right) .
$$

Последняя импликация вытекает из следующего сверхтождества (при $X=A_{1}$, $\left.Y=A_{2}, Z=A_{3}\right)$ :

$$
X(Y(x, y), Z(x, y))=X(X(Y(x, Z(x, y)), Y(x, y)), Z(x, y))
$$

которое выполняется в любой квазирешетке $\mathfrak{A}=\langle Q ; \Sigma\rangle$, поскольку оно является следствием сверхтождеств (3.1)-(3.4). Действительно, достаточно сверхтождество (1.2) записать в виде

$$
X(Y(x, y), z)=X(X(Y(x, z), Y(x, y)), z),
$$

и здесь вместо $z$ положить $Z(x, y)$.

В результате квазирешетка $\mathfrak{A}=\langle Q ; \Sigma\rangle$ оказывается изоморфной подпрямому произведению $\left\langle\varphi_{0} Q ; \tilde{\psi}_{0} \Sigma\right\rangle$ квазирешеток $\mathfrak{A} / \Theta_{1,2}, \mathfrak{A} / \Theta_{1,3}, \mathfrak{A} / \Theta_{2,3}$, проекции которой не являются изоморфизмами. Покажем, например, что первая проекция подпрямого произведения $\left\langle\varphi_{0} Q ; \tilde{\psi}_{0} \Sigma\right\rangle$ не является изоморфизмом. Поскольку $A_{1} \neq A_{2}$, то существуют $x, y \in Q$ такие, что $A_{1}(x, y) \neq A_{2}(x, y)$, где $x \neq y$. Однако $\left[A_{1}(x, y)\right]_{1,2}=\left[A_{2}(x, y)\right]_{1,2}$, ибо

$$
\begin{aligned}
& f_{1,2}\left(A_{1}(x, y), A_{2}(x, y)\right)=A_{1}(x, y), \\
& f_{1,2}\left(A_{2}(x, y), A_{1}(x, y)\right)=A_{2}(x, y) .
\end{aligned}
$$

Действительно, воспользуемся сверхтождеством

$$
X(X(x, y), Y(X(x, y), Y(x, y)))=X(x, y),
$$

являющимся следствием сверхтождества (1.3):

$$
\begin{aligned}
X(x, y) & \stackrel{(1.3)}{=} X(X(x, y), Y(x, y)) \\
& \stackrel{(1.3)}{=} X(X(X(x, y), Y(x, y)), Y(X(x, y), Y(x, y))) \\
& \stackrel{(1.3)}{=} X(X(x, y), Y(X(x, y), Y(x, y))) .
\end{aligned}
$$

Имеем

$$
f_{1,2}\left(A_{1}(x, y), A_{2}(x, y)\right)=A_{1}\left(A_{1}(x, y), A_{2}\left(A_{1}(x, y), A_{2}(x, y)\right)\right) \stackrel{(3.11)}{=} A_{1}(x, y) .
$$

Аналогично, с использованием сверхтождества

$$
Y(x, y) \stackrel{(3.1)}{=} X(Y(x, y), Y(x, y)) \stackrel{(1.3)}{=} X(Y(x, y), Y(Y(x, y), X(x, y)))
$$

доказываем второе равенство. Лемма 3.1 доказана. 
СлЕДСТВИЕ 3.1. Если бинарная алгебра $\mathfrak{A}=\langle Q ; \Sigma\rangle$ со сверхтождествами (3.1)-(3.3), (3.5)-(3.7), (3.9)-(3.12) подпрямо-неразложима в категории $T$-алгебр, где $T=\{2\}$, то мощность $|\Sigma|$ не больше 2.

СлЕДСТВИЕ 3.2. Если бинарная алгебра $\mathfrak{A}=\langle Q ; \Sigma\rangle$ со сверхтождествами (1.2), (3.1)-(3.3), (3.5), (3.9) подпрямо-неразложима в категории Т-алгебр, где $T=\{2\}$, то мощность $|\Sigma|$ не больше 2 .

СЛЕДСТВИЕ 3.3. Если бинарная алгебра $\mathfrak{A}=\langle Q ; \Sigma\rangle$ со сверхтождествами (1.2), (3.1)-(3.3), (3.5), (3.8) подпрямо-неразложима в категории Т-алгебр, где $T=\{2\}$, то мощность $|\Sigma|$ не больше 2 .

ЗАмечАниЕ 3.1 . Система сверхтождеств $(1.2),(3.1)-(3.3),(3.5),(3.8)$ избыточна. Сверхтождества (1.2), (3.5) являются следствиями сверхтождеств (3.1)-(3.3), (3.8). Покажем, например, что сверхтождество (1.2) является следствием сверхтождеств (3.1)-(3.3), (3.8). Согласно сверхтождеству (3.8) имеем

$$
\begin{gathered}
X(Y(X(Y(z, y), x), X(y, x)), Y(x, X(y, x))) \\
=Y(X(Y(z, y), x), X(y, x)) \\
X(Y(X(x, y), x), Y(y, x))=Y(X(x, y), x) .
\end{gathered}
$$

Следовательно,

$$
\begin{aligned}
Y(X(Y(z, y), x), X(y, x)) \\
\quad \stackrel{(3.13)}{=} X(Y(X(Y(z, y), x), X(y, x)), Y(x, X(y, x))) \\
\quad \stackrel{(3.14)}{=} X(Y(X(Y(z, y), x), X(y, x)), X(Y(X(x, y), x), Y(y, x))) \\
\quad \stackrel{(3.13)}{=} X(Y(X(Y(z, y), x), X(y, x)), Y(y, x))
\end{aligned}
$$

и

$$
\begin{aligned}
& X(x, X(Y(x, y), Y(y, z)))=X(X(Y(z, y), x), Y(y, x)) \\
& \stackrel{(3.8)}{=} X(Y(X(Y(z, y), x), X(y, x)), Y(y, x)) \\
& \quad=Y(X(Y(z, y), x), X(y, x)) \stackrel{(3.8)}{=} X(Y(z, y), x) .
\end{aligned}
$$

ЛЕмма 3.2. В категории Т-алгебр, где $T=\{2\}$, подпрямо-неразложимые а-квазирешетки являются дистрибутивными решетками.

ДокАЗАТЕЛЬСТво. Пусть $\mathfrak{A}=\langle Q ; \Sigma\rangle$ является $a$-квазирешеткой, т.е. квазирешеткой с дополнительным условием

$$
A_{1}\left(x, \ldots A_{n-1}\left(x, A_{n}(x, y)\right) \ldots\right)=x,
$$

где $A_{1}, \ldots, A_{n-1}, A_{n} \in \Sigma$. Если $\mathfrak{A}$ подпрямо-неразложима, то согласно лемме 3.1 множество операций $\Sigma$ содержит не более двух элементов. Пусть $A_{1}=\cdots$ $\cdots=A_{n-1}=A_{n}$; тогда $A_{1}(x, y)=x$ и $A_{1}(x, y) \neq A_{1}(y, x)$, что противоречит 
условию (3.2) определения квазирешетки $(|Q| \neq 1)$. Поэтому $|\Sigma|=2$, и (поскольку равенство операций $A_{1}=\cdots=A_{n-1}=A_{n}$ исключается, то) допустим $A_{i_{1}}=\cdots=A_{i_{k}}=(\cdot)$ и $A_{i_{k+1}}=\cdots=A_{i_{n}}=(+)$, где $i_{1}, \ldots, i_{n}$ - перестановка чисел $1, \ldots, n$. Тогда согласно сверхтождествам (3.4) и (3.1) имеем

$$
\begin{aligned}
& A_{1}\left(x, \ldots A_{n-1}\left(x, A_{n}(x, y)\right) \ldots\right) \\
& \quad=A_{i_{1}}\left(x, \ldots A_{i_{k}}\left(x, A_{i_{k+1}}\left(x, \ldots A_{i_{n}}(x, y) \ldots\right)\right) \ldots\right)=x
\end{aligned}
$$

т.е.

$$
x(x+y)=x,
$$

и подпрямо-неразложимая $a$-квазирешетка $\mathfrak{A}=\langle Q ; \Sigma\rangle$ будет решеткой.

СлеДСТвИЕ 3.4. В категории T-алгебр, где $T=\{2\}$, любая а-квазирешетка $\mathfrak{A}=\langle Q ; \Sigma\rangle$ изоморфна подпрямому произведению дистрибутивных решеток.

СледСТвиЕ 3.5. В каждой а-квазирешетке $\mathfrak{A}=\langle Q ; \Sigma\rangle$ выполняется любое сверхтождество многообразия дистрибутивных решеток.

Пусть $\mathfrak{A}$ - $T$-алгебра. Главные подсистемы $\left\langle Q_{i} ; \Sigma\right\rangle, i \in I$, алгебры $\mathfrak{A}$ называются дизбюнктными, если $Q_{i} \cap Q_{j}=\varnothing$ для любых $i, j \in I, i \neq j$.

ОПРЕДЕлЕНИЕ 3.2. $T$-алгебра $\mathfrak{A}=\langle Q ; \Sigma\rangle$ называется сумм.й своих дизбюнктных подсистем $\left\langle Q_{i} ; \Sigma\right\rangle, i \in I$, если $Q=\bigcup_{i \in I} Q_{i}$ и на множестве индексов $I$ можно определить частичный порядок "₹" так, что вьполняются следуюшие условия (см. [35]):

1) $I(\leqslant)$ - верхняя полурешетка;

$2)$ если $i \leqslant j$, то существует гомоморфизм $\left(\varphi_{i, j}, \tilde{\varepsilon}\right):\left\langle Q_{i} ; \Sigma\right\rangle \Longrightarrow\left\langle Q_{j} ; \Sigma\right\rangle$, причем $\varphi_{i, i}=\varepsilon-$ тождественное отображение и $\varphi_{i, j} \cdot \varphi_{j, k}=\varphi_{i, k}$, где $i \leqslant j \leqslant k$;

3 для любой операции $A \in \Sigma,|A|=n$, и для любых $x_{1}, \ldots, x_{n} \in Q$

$$
A\left(x_{1}, \ldots, x_{n}\right)=A\left(\varphi_{i_{1}, i_{0}}\left(x_{1}\right), \ldots, \varphi_{i_{n}, i_{0}}\left(x_{n}\right)\right),
$$

где $x_{1} \in Q_{i_{1}}, \ldots, x_{n} \in Q_{i_{n}}, i_{1}, \ldots, i_{n} \in I, i_{0}=i_{1}+\cdots+i_{n}$.

Сверхтождества нетривиальных решеток - однородные сверхтождества, а если в дизъюнктных подсистемах $\left\langle Q_{i} ; \Sigma\right\rangle, i \in I$ (как подалгебрах), выполняется одно и то же однородное сверхтождество, то, очевидно, оно будет выполняться и в их сумме $\mathfrak{A}=\langle Q ; \Sigma\rangle$. Напомним также понятие $P$-функции, введенной в [35].

Функция $f: Q \times Q \rightarrow Q$ называется $P$-функцией для $T$-алгебры $\mathfrak{A}=\langle Q ; \Sigma\rangle$, если выполняются следующие тождества:

$$
\begin{aligned}
f(x, x) & =x, \\
f(x, f(y, z)) & =f(f(x, y), z), \\
f(x, f(y, z)) & =f(x, f(z, y)), \\
f\left(F\left(x_{1}, \ldots, x_{n}\right), y\right) & =F\left(f\left(x_{1}, y\right), \ldots, f\left(x_{n}, y\right)\right), \\
f\left(y, F\left(x_{1}, \ldots, x_{n}\right)\right) & =f\left(y, F\left(f\left(y, x_{1}\right), \ldots, f\left(y, x_{n}\right)\right)\right), \\
f\left(F\left(x_{1}, \ldots, x_{n}\right), x_{k}\right) & =F\left(x_{1}, \ldots, x_{n}\right), \quad 1 \leqslant k \leqslant n, \\
f(y, F(y, \ldots, y)) & =y
\end{aligned}
$$


для любого $n$-арного $F \in \Sigma, n \in T$. Если для $T$-алгебры $\mathfrak{A}=\langle Q ; \Sigma\rangle$ существует $P$-функция $f$, то либо $\mathfrak{A}$ удовлетворяет условию $f(x, y)=x$ для любых $x, y \in Q$, либо сушествует представление $\mathfrak{A}$ в виде суммы дизъюнктных подсистем $\left\langle Q_{i} ; \Sigma\right\rangle$, $i \in I$, с условием $f(x, y)=x$ для любых $x, y \in Q_{i}, i \in I$ (см. [35]).

ЛЕмма 3.3 [33]. Любая квазирешетка с двумя бинарными операциями либо является дистрибутивной решеткой, либо суммой своих дизвюнктных подсистем, являющихся дистрибутивными решетками.

ДоКАЗАТЕЛЬСТВО. Функция $f(x, y)=x+x y$ является $P$-функцией для любой квазирешетки $Q(+, \cdot)$.

ЛЕмма 3.4 [34]. Любая бинарная алгебра с двумя бинарными операциями и со сверхтождествами (3.1)-(3.3), (3.8) либо является решеткой, либо суммой своих дизвюнктных подсистем, являющихся решетками.

ДокАЗАТЕЛЬСТво. Функция $f(x, y)=x+x y$ является $P$-функцией для любой бинарной алгебры $Q(+, \cdot)$ со сверхтождествами $(3.1)-(3.3),(3.8)$.

ЛЕмма 3.5. Любая бинарная алгебра с двумя бинарными операчиями и со сверхтохсдествами (1.1), (3.1)-(3.3), (3.8) либо является модулярной решеткой, либо суммой своих дизбюнктных подсистем, являющихся модулярныци решетками.

Доказательство вытекает из леммы 3.4 и примера 1.4.

ДОКАЗАТЕЛЬСТВО ТЕОРЕМЫ 3.1. Согласно лемме 3.1 в категории $T$-алгебр любая квазирешетка $\mathfrak{A}=\langle Q ; \Sigma\rangle$ изоморфна подпрямому произведению квазирешеток с одной или двумя бинарными операциями. В любой квазирешетке с одной бинарной операцией (т.е. в полурешетке), очевидно, выполняется любое однородное сверхтождество (тождество). В любой квазирешетке с двумя бинарными операциями согласно лемме 3.3 выполняется любое сверхтождество многообразия дистрибутивных решеток.

ТЕОРема 3.2. Любое сверхтождество многообразия модулярных решеток является следствием сверхтождеств (1.1), (1.2), (3.1)-(3.3), (3.5), (3.8) (см. также замечание 3.1).

ДоКАЗАТЕЛЬСТво. Повторяется доказательство теоремы 3.1 с применением следствия 3.3 и леммы 3.5 .

ТЕОРема 3.3. Любое сверхтождество многообразия решеток является следствием сверхтождеств (1.2), (3.1)-(3.3), (3.5), (3.8) (см. также замечание 3.1$)$.

ДоКАЗАТЕЛЬСТво. Повторяется доказательство теоремы 3.1 с использованием следствия 3.3 и леммы 3.4 .

Поскольку многообразие дистрибутивных решеток не имеет собственных подмногообразий, то из теоремы 3.1 вытекает характеризация сверхтождеств произвольной отдельно взятой дистрибутивной решетки.

СЛЕДСТВИЕ 3.6. Любое сверхтождество нетривиальной дистрибутивной решетки является следствием сверхтождеств (3.1)-(3.4). 
ДоКАЗАТЕЛЬСТво. Пусть в дистрибутивной решетке $L$ вьполняется сверхтождество $(\mathrm{h})$, которое равносильно конечной системе тождеств $\left(\mathrm{h}_{1}\right), \ldots,\left(\mathrm{h}_{m}\right)$, выполняюшихся в $L$. Поэтому в любой дистрибутивной решетке выполняются тождества $\left(\mathrm{h}_{1}\right), \ldots,\left(\mathrm{h}_{m}\right)$. Следовательно, в любой дистрибутивной решетке выполняется сверхтождество (h), т.е. (h) - сверхтождество многообразия дистрибутивных решеток, и потому согласно теореме 3.1 является следствием сверхтождеств (3.1)-(3.4).

\section{§4. Алгебры со сверхтождествами и} регулярными тождествами булевых алгебр

ТЕОРема 4.1 [13]. Любое сверхтождество многообразия булевых алгебр является следствием следуюших одиннадиати ${ }^{4}$ сверхтождеств: (3.1)-(3.4), a также

$$
\begin{aligned}
& F(x)=G(x) \quad(=\bar{x}), \\
& \overline{\bar{x}}=x, \\
& X(x, \bar{x})=X(y, \bar{y}), \\
& X(x, \overline{X(y, \bar{y})})=x, \\
& \overline{X(\overline{Y(x, y)}, z)}=Y(\overline{X(\bar{x}, z)}, \overline{X(\bar{y}, z)}), \\
& X(Y(x, y), Y(x, z))=X(Y(\overline{Y(\bar{z}, \bar{y})}, x), Y(x, Y(y, z))), \\
& X(\overline{X(x, Y(x, y))}, \overline{X(z, Y(z, u))}) \\
& =X(\overline{X(Y(x, z), Y(u, \bar{u}))}, \overline{X(\overline{Y(u, \bar{u})}, \overline{Y(\overline{Y(x, y)}, \overline{Y(z, u)})})}) .
\end{aligned}
$$

Поскольку многообразие булевых алгебр не имеет собственных подмногообразий, то отсюда вытекает характеризация сверхтождеств произвольной булевой алгебры.

СледСТВИЕ 4.1. Любое сверхтождество булевой алгебры является следствием сверхтождеств (3.1)-(3.4), (4.1)-(4.7).

ДокАЗАТЕльСтво. См. доказательство следствия 3.6.

Из теоремы 4.1 в качестве следствия получаем также характеризацию регулярных тождеств булевых алгебр.

Тождество булевой алгебры $\mathfrak{A}=Q\left(+, \cdot,^{-}\right)$называется регулярныц, если оно остается истинным в $\mathfrak{A}$ при замене $(\cdot) \rightarrow(+)$. Например, тождество

$$
\overline{x+y}=\bar{x} \cdot \bar{y}
$$

\footnotetext{
${ }^{4}$ Сверхтождество 9) из [13], являясь следствием сверхтождеств (3.1)-(3.4) (согласно теореме 3.1 , здесь опускается. Кроме того, в последнем сверхтождестве (4.7) вместо терма $Y(v, \bar{v})$ записан $Y(u, \bar{u})$ (согласно сверхтождеству $(4.3))$.
} 
не регулярно, а тождество

$$
\overline{x+\overline{y \cdot \bar{y}}}=\bar{x} \cdot \overline{y+\bar{y}}
$$

является регулярным. Регулярными считаются и те тождества, в которых не участвует операция (·). С учетом принщипа двойственности приходим к следующему результату.

СЛЕДСТВИЕ 4.2. Любое регулярное тождество булевой алгебры (или многообразия булевых алгебр) является следствием конечной системы тождеств, полученных из сверхтождеств (3.1)-(3.4), (4.2)-(4.7) при заменах $X \rightarrow(+),(\cdot)$ u $Y \rightarrow(+),(\cdot)$.

Алгебра $\mathfrak{B}$ с двумя бинарными и одной унарной операциями, удовлетворяюшая сверхтождествам многообразия булевых алгебр, вообще говоря, не является булевой алгеброй. Действительно, в любой булевой алгебре $\mathfrak{A}=Q\left(+, \cdot{ }^{-}\right)$выполняются сверхтождества (3.1)-(3.4), (4.1)-(4.7). Алгебра $\mathfrak{A}^{\prime}=\mathfrak{A} \times \mathfrak{A}$, как прямое произведение $T$-алгебр, где $T=\{1,2\}$, будет $T$-алгеброй с пятью операциями:

$$
A=(+,+), \quad B=(+, \cdot), \quad C=(\cdot,+), \quad D=(\cdot, \cdot), \quad E=\left({ }^{-},{ }^{-}\right) .
$$

В этой алгебре $\mathfrak{A}^{\prime}=\langle Q \times Q ;\{A, B, C, D, E\}\rangle$ также выполняются указанные сверхтождества. Однако непосредственная проверка показывает, что в ее подалгебре $\langle Q \times Q ;\{A, B\}\rangle$ не выполняются тождества поглошения, поэтому алгебра $\langle Q \times Q ;\{A, B, E\}\rangle$ не является булевой алгеброй, хотя в ней выполняются все сверхтождества многообразия булевых алгебр.

Полное описание алгебр с двумя бинарными и одной унарной операциями, удовлетворяющих сверхтождествам многообразия булевых алгебр, дано в теореме 4.2 (см. ниже).

ОПРЕДЕЛЕНИЕ 4.1. $T$-алгебру $\mathfrak{A}=\langle Q ; \Sigma\rangle$, где $T=\{1,2\}$, назовем булевой квазирешеткой, если она удовлетворяет сверхтождествам многообразия булевых алгебр, т.е. сверхтождествам (3.1)-(3.4) (из §3) и (4.1)-(4.7).

ПримеР 4.1. Прямое $T$-произведение булевых алгебр, где $T=\{1,2\}$, является булевой квазирешеткой.

ЛЕмма 4.1. Если булева квазирешетка $\mathfrak{A}=\langle Q ; \Sigma\rangle$ подпрямо-неразложима в категории $T$-алгебр, где $T=\{1,2\}$, то мощность множества ее бинарных операчий не больие 2.

ДокАЗАТЕЛЬСтво. К доказательству леммы 3.1 достаточно здесь добавить, что отношения $\Theta_{i, j}$, где $i, j=1,2,3$ и $i<j$, согласованы и с унарной операцией “-” булевой квазирешетки $\mathfrak{A}=\langle Q ; \Sigma\rangle$ :

$$
x \Theta_{i, j} y \Longrightarrow \bar{x} \Theta_{i, j} \bar{y}, \quad x, y \in Q
$$

Действительно, в любой булевой алгебре (т.е. в многообразии булевых алгебр) вьполняется сверхтождество

$$
X(\overline{X(x, Y(x, y))}, Y(\overline{X(x, Y(x, y))}, \overline{X(y, Y(y, x))}))=\overline{X(x, Y(x, y))},
$$


поэтому это сверхтождество выполняется и в любой булевой квазирешетке $\mathfrak{A}=$ $\langle Q ; \Sigma\rangle$. Следовательно,

$$
\begin{aligned}
f_{i, j}(\bar{x}, \bar{y}) & =A_{i}\left(\bar{x}, A_{j}(\bar{x}, \bar{y})\right) \\
& =A_{i}\left(\overline{A_{i}\left(x, A_{j}(x, y)\right)}, A_{j}\left(\overline{A_{i}\left(x, A_{j}(x, y)\right)}, \overline{A_{i}\left(y, A_{j}(y, x)\right)}\right)\right) \\
& \stackrel{(4.8)}{=} \overline{A_{i}\left(x, A_{j}(x, y)\right)}=\bar{x} .
\end{aligned}
$$

Аналогично получаем равенство

$$
f_{i, j}(\bar{y}, \bar{x})=\bar{y}
$$

Итак, $\bar{x} \Theta_{i, j} \bar{y}$, и отношение $\Theta_{i, j}$ является конгруэнцией для булевой квазирешетки $\mathfrak{A}$.

СледСтвиЕ 4.3. В категории $T$-алгебр, где $T=\{1,2\}$, любая булева квазирешетка $\mathfrak{A}=\langle Q ; \Sigma\rangle$ изоморфна подпрямому произведения булевых квазирешеток с одной или двумя бинарными операциями.

Итак, описание булевых квазирешеток сводится к описаниям булевых квазирешеток с одной или двумя бинарными операциями.

В случае одной бинарной операции сверхтождества (3.1)-(3.4), (4.1)-(4.7) булевой квазирешетки преврашается в тождества, причем эта система тождеств избыточна. Например, тождества, соответствующие сверхтождествам (4.6) и (4.7), следуют из остальных (см. также следствие 1.2 из [13]). Таким образом, булева квазирешетка с одной бинарной операцией - алгебра $Q\left(+,{ }^{-}\right)$со следующими тождествами:

$$
\begin{gathered}
x+x=x, \\
x+y=y+x, \\
x+(y+z)=(x+y)+z, \\
\overline{\bar{x}}=x, \\
x+\bar{x}=y+\bar{y}, \\
x+(\overline{y+\bar{y}})=x, \\
\overline{\overline{x+y}+z}=\overline{\bar{x}+z}+\overline{\bar{y}+z} .
\end{gathered}
$$

Очевидно, что если $Q\left(+, \cdot,{ }^{-}\right)$- булева алгебра, то подалгебра (редукт) $Q\left(+,{ }^{-}\right)-$ булева квазирешетка. Верно и обратное, если $Q\left(+,{ }^{-}\right)$- булева квазирешетка, то, определяя на множестве $Q$ умножение

$$
x \cdot y=\overline{\bar{x}+\bar{y}}
$$

получим булеву алгебру $Q\left(+, \cdot,^{-}\right)$. 
Остается характеризация булевых квазирешеток с двумя бинарными операциями. Представление булевой квазирешетки в виде суммы дизъюнктных подсистем невозможно, поскольку в булевой квазирешетке выполняются сверхтождества (следовательно, и тождества), не являющиеся однородными.

Для характеризации булевых квазирешеток с двумя бинарными операциями введем следуюшее понятие булевой суммы и докажем, что любая булева квазирешетка с двумя бинарными операциями является булевой суммой булевых алгебр.

Пусть $\mathfrak{A}=\langle Q ; \Omega \cup\{F\}\rangle-T$-алгебра с одной унарной операцией $F$. Пусть $\left\langle Q_{i} ; \Omega\right\rangle, i \in I,-$ подсистемы (подалгебры) для алгебры $\mathfrak{A}$, и пусть $\mathfrak{A}_{i}=\left\langle Q_{i} ; \Omega \cup\right.$ $\left.\left\{F_{i}\right\}\right\rangle$ - алгебра с одной унарной операцией $F_{i}$ для любого $i \in I$. Алгебра $\mathfrak{A}=$ $\langle Q ; \Omega \cup\{F\}\rangle$ называется булевой суммой алгебр $\mathfrak{A}_{i}=\left\langle Q_{i} ; \Omega \cup\left\{F_{i}\right\}\right\rangle, i \in I$, если:

а) $Q_{i} \cap Q_{j}=\varnothing$, где $i, j \in I, i \neq j$;

б) $Q=\bigcup_{i \in I} Q_{i}$

в) на множестве индексов $I$ можно определить частичный порядок "\$” так, что $I(\leqslant)$ - булева алгебра;

г) если $i \leqslant j$, то сушествует изоморфизм

$$
\left(\varphi_{i, j}, \tilde{\varepsilon}\right): \mathfrak{A}_{i} \Longrightarrow \mathfrak{A}_{j}, \quad i, j \in I
$$

где $\tilde{\varepsilon}\left(F_{i}\right)=F_{j}, \tilde{\varepsilon}(A)=A$ для любого $A \in \Omega$, причем $\varphi_{i, i}=\varepsilon$ и $\varphi_{i, j} \cdot \varphi_{j, k}=\varphi_{i, k}$, где $i \leqslant j \leqslant k$;

д) для любого $i \in I$ сушествует изоморфизм

$$
\left(h_{i, \bar{i}}, \tilde{\varepsilon}\right): \mathfrak{A}_{i} \Longrightarrow \mathfrak{A}_{\bar{i}},
$$

причем $h_{i, \bar{i}}^{-1}=h_{\bar{i}, i}$ и $\varphi_{i, 1}=h_{i, \bar{i}} \cdot \varphi_{\bar{i}, 1}$, где 1 - единица булевой алгебры $I(\leqslant)$, а $\bar{i}-$ дополнение в этой решетке;

е) для любого $A \in \Omega,|A|=n \geqslant 2$, и для любых $x_{1}, \ldots, x_{n} \in Q$

$$
A\left(x_{1}, \ldots, x_{n}\right)=A\left(\varphi_{i_{1}, i_{0}}\left(x_{1}\right), \ldots, \varphi_{i_{n}, i_{0}}\left(x_{n}\right)\right)
$$

где $x_{1} \in Q_{i_{1}}, \ldots, x_{n} \in Q_{i_{n}}, i_{1}, \ldots, i_{n} \in I, i_{0}=i_{1}+\cdots+i_{n}$;

ж) для унарной операции $F$ и для любого $x \in Q$

$$
F(x)=h_{i, \bar{i}}\left[F_{i}(x)\right]
$$

где $x \in Q_{i}$.

TеОрема 4.2. Алгебра $\mathfrak{A}=Q(+, \cdot,-)$ с двумя бинарными и одной унарной операциями удостоверяет сверхтожсдествам многообразия булевых алгебр (т.е. является булевой квазирешеткой) тогда и только тогда, когда $\mathfrak{A}$ является булевой алгеброй или булевой суммой булевых алгебр.

Предварительно докажем одно утверждение о значениях булевых слов (термов). Здесь булево слово - это слово от (не более чем) двух символов бинарных операций "+”, “.” и одного символа унарной операции. Если $\omega$ - булево слово от предметных переменных $x_{1}, \ldots, x_{n}$, то обозначим $\omega=\omega\left(x_{1}, \ldots, x_{n}\right)$. 
ЛЕмма 4.2. Пусть $\omega=\omega\left(x_{1}, \ldots, x_{n}\right)$ - булево слово. Если алгебра $\mathfrak{A}=Q\left(+, \cdot,{ }^{-}\right)$является булевой суммой булевых алгебр $Q_{i}, \quad i \in I$, то при значениях $x_{1}=a_{1} \in Q_{i_{1}}, \ldots, x_{n}=a_{n} \in Q_{i_{n}}$ имеем $\omega\left(a_{1}, \ldots, a_{n}\right) \in Q_{k}$, где $k=\omega\left(i_{1}, \ldots, i_{n}\right)$ - значение $\omega\left(x_{1}, \ldots, x_{n}\right)$ в булевой алгебре $I=I\left(+, \cdot,{ }^{-}\right)$при $x_{1}=i_{1} \in I, \ldots, x_{n}=i_{n} \in I$. Более того,

$$
\omega\left(\varphi_{i_{1}, 1}\left(a_{1}\right), \ldots, \varphi_{i_{n}, 1}\left(a_{n}\right)\right)=\varphi_{k, 1}\left(\omega\left(a_{1}, \ldots, a_{n}\right)\right) .
$$

ДокАЗАТЕЛЬСТВо (по индукции). Если $\omega=x$, то утверждение очевидно. Пусть $\omega=\omega_{1}+\omega_{2}$, и пусть для $\omega_{1}$ и $\omega_{2}$ утверждение доказано. Без ушерба обшности можно предположить, что $\omega=\omega\left(x_{1}, \ldots, x_{n}\right), \omega_{1}=\omega_{1}\left(x_{1}, \ldots, x_{n}\right)$, $\omega_{2}=\omega_{2}\left(x_{1}, \ldots, x_{n}\right)$. Тогда по предположению индукции

$$
\begin{aligned}
& \omega_{1}\left(\varphi_{i_{1}, 1}\left(a_{1}\right), \ldots, \varphi_{i_{n}, 1}\left(a_{n}\right)\right)=\varphi_{k_{1}, 1}\left(\omega_{1}\left(a_{1}, \ldots, a_{n}\right)\right), \\
& \omega_{2}\left(\varphi_{i_{1}, 1}\left(a_{1}\right), \ldots, \varphi_{i_{n}, 1}\left(a_{n}\right)\right)=\varphi_{k_{2}, 1}\left(\omega_{2}\left(a_{1}, \ldots, a_{n}\right)\right),
\end{aligned}
$$

где $k_{1}=\omega_{1}\left(i_{1}, \ldots, i_{n}\right), k_{2}=\omega_{2}\left(i_{1}, \ldots, i_{n}\right), \omega_{1}\left(a_{1}, \ldots, a_{n}\right) \in Q_{k_{1}}, \omega_{2}\left(a_{1}, \ldots, a_{n}\right) \in$ $Q_{k_{2}}$. Поскольку

$$
k=\omega\left(i_{1}, \ldots, i_{n}\right)=\omega_{1}\left(i_{1}, \ldots, i_{n}\right)+\omega_{2}\left(i_{1}, \ldots, i_{n}\right)=k_{1}+k_{2},
$$

Tо

$$
\begin{aligned}
& \omega\left(\varphi_{i_{1}, 1}\left(a_{1}\right), \ldots, \varphi_{i_{n}, 1}\left(a_{n}\right)\right)=\omega_{1}\left(\varphi_{i_{1}, 1}\left(a_{1}\right), \ldots, \varphi_{i_{n}, 1}\left(a_{n}\right)\right) \\
& \quad+\omega_{2}\left(\varphi_{i_{1}, 1}\left(a_{1}\right), \ldots, \varphi_{i_{n}, 1}\left(a_{n}\right)\right)=\varphi_{k_{1}, 1}\left(\omega_{1}\left(a_{1}, \ldots, a_{n}\right)\right)+\varphi_{k_{2}, 1}\left(\omega_{2}\left(a_{1}, \ldots, a_{n}\right)\right) \\
& \quad=\varphi_{k, 1}\left(\varphi_{k_{1}, k}\left(\omega_{1}\left(a_{1}, \ldots, a_{n}\right)\right)\right)+\varphi_{k, 1}\left(\varphi_{k_{2}, k}\left(\omega_{2}\left(a_{1}, \ldots, a_{n}\right)\right)\right) \\
& \quad=\varphi_{k, 1}\left(\varphi_{k_{1}, k}\left(\omega_{1}\left(a_{1}, \ldots, a_{n}\right)\right)+\varphi_{k_{2}, k}\left(\omega_{2}\left(a_{1}, \ldots, a_{n}\right)\right)\right) \\
& \quad=\varphi_{k, 1}\left(\omega_{1}\left(a_{1}, \ldots, a_{n}\right)+\omega_{2}\left(a_{1}, \ldots, a_{n}\right)\right)=\varphi_{k, 1}\left(\omega\left(a_{1}, \ldots, a_{n}\right)\right),
\end{aligned}
$$

где $\omega\left(a_{1}, \ldots, a_{n}\right) \in Q_{k}$.

Аналогично рассматривается случай $\omega=\omega_{1} \cdot \omega_{2}$.

Пусть $\omega\left(x_{1}, \ldots, x_{n}\right)=\overline{\pi\left(x_{1}, \ldots, x_{n}\right)}$, и пусть

$$
\pi\left(\varphi_{i_{1}, 1}\left(a_{1}\right), \ldots, \varphi_{i_{n}, 1}\left(a_{n}\right)\right)=\varphi_{\bar{k}, 1}\left(\pi\left(a_{1}, \ldots, a_{n}\right)\right),
$$

где $\bar{k}=\pi\left(i_{1}, \ldots, i_{n}\right)$ и $\pi\left(a_{1}, \ldots, a_{n}\right) \in Q_{\bar{k}}$. Тогда

$$
\begin{aligned}
& \omega\left(\varphi_{i_{1}, 1}\left(a_{1}\right), \ldots, \varphi_{i_{n}, 1}\left(a_{n}\right)\right)=\left(\pi\left(\varphi_{i_{1}, 1}\left(a_{1}\right), \ldots, \varphi_{i_{n}, 1}\left(a_{n}\right)\right)\right)^{\prime} \\
& \quad=\left(\varphi_{\bar{k}, 1}\left(\pi\left(a_{1}, \ldots, a_{n}\right)\right)\right)^{\prime}=\varphi_{\bar{k}, 1}\left(\left(\pi\left(a_{1}, \ldots, a_{n}\right)\right)^{\prime}\right) \\
& \quad=\varphi_{k, 1}\left(h_{\bar{k}, k}\left(\left(\pi\left(a_{1}, \ldots, a_{n}\right)\right)^{\prime}\right)\right)=\varphi_{k, 1}\left(\overline{\pi\left(a_{1}, \ldots, a_{n}\right)}\right) \\
& \quad=\varphi_{k, 1}\left(\omega\left(a_{1}, \ldots, a_{n}\right)\right),
\end{aligned}
$$

где $\omega\left(a_{1}, \ldots, a_{n}\right) \in Q_{k}, \bar{k}=\overline{\pi\left(i_{1}, \ldots, i_{n}\right)}=\omega\left(i_{1}, \ldots, i_{n}\right)$. Лемма 4.2 доказана. 
ДОКАЗАТЕЛЬСТВО ТЕОРЕМЫ 4.2. ДОСТАТОчНОСТь. Пусть алгебра $\mathfrak{A}=$ $Q\left(+, \cdot,^{-}\right)$с двумя бинарными и одной унарной операциями является булевой суммой булевых алгебр $Q_{i}, i \in I$, и пусть $w_{1}=w_{2}$ - произвольное сверхтождество многообразия булевых алгебр. Покажем, что это сверхтождество выполняется и в алгебре $\mathfrak{A}$. При замене бинарных функциональных переменных бинарными операциями алгебры $\mathfrak{A}$ равенство $w_{1}=w_{2}$ преврашается в тождество (булевых слов), выполняющееся в любой булевой алгебре. Требуется показать, что это тождество вьполняется и в алгебре $\mathfrak{A}$.

Без ограничения общности можно предположить, что $w_{1}=w_{1}\left(x_{1}, \ldots, x_{n}\right)$ и $w_{2}=w_{2}\left(x_{1}, \ldots, x_{n}\right)$. Согласно лемме 4.2 имеем

$$
\begin{aligned}
& w_{1}\left(\varphi_{i_{1}, 1}\left(a_{1}\right), \ldots, \varphi_{i_{n}, 1}\left(a_{n}\right)\right)=\varphi_{k_{1}, 1}\left(w_{1}\left(a_{1}, \ldots, a_{n}\right)\right), \\
& w_{2}\left(\varphi_{i_{1}, 1}\left(a_{1}\right), \ldots, \varphi_{i_{n}, 1}\left(a_{n}\right)\right)=\varphi_{k_{2}, 1}\left(w_{2}\left(a_{1}, \ldots, a_{n}\right)\right),
\end{aligned}
$$

где $k_{1}=w_{1}\left(i_{1}, \ldots, i_{n}\right), \quad k_{2}=w_{2}\left(i_{1}, \ldots, i_{n}\right)$. Поскольку тождество $w_{1}\left(x_{1}, \ldots\right.$ $\left.\ldots, x_{n}\right)=w_{2}\left(x_{1}, \ldots, x_{n}\right)$ выполняется и в булевой алгебре $I$, то $k_{1}=k_{2}=k$. Кроме того, тождество $w_{1}\left(x_{1}, \ldots x_{n}\right)=w_{2}\left(x_{1}, \ldots, x_{n}\right)$ выполняется и в булевой алгебре $Q_{1}$, где 1 - единица булевой алгебры $I$. Поэтому

$$
w_{1}\left(\varphi_{i_{1}, 1}\left(a_{1}\right), \ldots, \varphi_{i_{n}, 1}\left(a_{n}\right)\right)=w_{2}\left(\varphi_{i_{1}, 1}\left(a_{1}\right), \ldots, \varphi_{i_{n}, 1}\left(a_{n}\right)\right) .
$$

Следовательно,

$$
\varphi_{k, 1}\left(w_{1}\left(a_{1}, \ldots, a_{n}\right)\right)=\varphi_{k, 1}\left(w_{2}\left(a_{1}, \ldots, a_{n}\right)\right)
$$

и согласно инъективности $\varphi_{k, 1}$

$$
w_{1}\left(a_{1}, \ldots, a_{n}\right)=w_{2}\left(a_{1}, \ldots, a_{n}\right)
$$

для любых $a_{1}, \ldots, a_{n} \in Q=\bigcup_{i \in I} Q_{i}$.

НЕобходимость. Требуется доказать, что любая булева квазирешетка $\mathfrak{A}=$ $Q\left(+, \cdot,^{-}\right)$с двумя бинарными операциями является булевой суммой булевых алгебр. При доказательстве, помимо сверхтождества (4.8), воспользуемся и следуюшими сверхтождествами многообразия булевых алгебр:

$$
\begin{gathered}
X(x, Y(x, X(Y(x, \overline{X(x, \bar{x})}), \overline{Y(x, X(x, \bar{x}))})))=x \\
X(X(Y(x, \overline{X(x, \bar{x})}), \overline{Y(x, X(x, \bar{x}))}), Y(x, X(Y(x, \overline{X(x, \bar{x})}), \overline{Y(x, X(x, \bar{x}))}))) \\
=X(Y(x, \overline{X(x, \bar{x})}), \overline{Y(x, X(x, \bar{x}))}) \\
X(Y(y, \overline{X(y, \bar{y})}), \overline{Y(y, X(y, \bar{y}))})=x
\end{gathered}
$$

где через $y$ обозначен терм

$$
X(Y(x, \overline{X(x, \bar{x})}), \overline{Y(x, X(x, \bar{x}))}) .
$$


Далее,

$$
\begin{aligned}
& X(Y(X(x, y), \overline{X(X(x, y), \overline{X(x, y)})}), \overline{Y(X(x, y), X(X(x, y), \overline{X(x, y)})})) \\
& =Y(X(Y(x, \overline{X(x, \bar{x})}), \overline{Y(x, X(x, \bar{x}))}), X(Y(y, \overline{X(y, \bar{y})}), \overline{Y(y, X(y, \bar{y}))})), \\
& X(x, Y(y, X(Y(y, \overline{X(y, \bar{y})}), \overline{Y(y, X(y, \bar{y}))})))=X(x, Y(x, y)), \\
& X(z, Y(y, \overline{X(x, \bar{x})}))=X(z, Y(z, y)) \\
& Y(Y(z, y), \overline{X(x, \bar{x})})=Y(X(y, Y(y, z)), \overline{X(x, \bar{x})}), \\
& X(z, X(y, \overline{Y(x, \bar{x})}))=X(X(y, Y(y, z)), \overline{Y(x, \bar{x})}), \\
& Y(z, X(y, \overline{Y(x, \bar{x})}))=X(z, Y(z, y)), \\
& \overline{X(y, \overline{Y(x, \bar{x})})}=Y(\bar{y}, \overline{X(x, \bar{x})}), \\
& X(X(x, y), Y(X(x, y), X(u, v)))=X(X(x, Y(x, u)), X(y, Y(y, v))) \text {, } \\
& X(Y(x, y), Y(Y(x, y), X(x, y)))=Y(x, y), \\
& \overline{X(\bar{x}, \overline{X(y, z)})}=X(\overline{X(\bar{x}, \bar{y})}, \overline{X(\bar{x}, \bar{z})}) \text {, } \\
& Y(X(Y(x, \overline{X(x, \bar{x})}), \overline{Y(x, X(x, \bar{x}))}), \\
& X(Y(x, y), X(Y(Y(x, y), \overline{X(Y(x, y), \overline{Y(x, y)})}), \overline{Y(Y(x, y), X(Y(x, y), \overline{Y(x, y)}))}) \\
& =X(Y(Y(x, y), \overline{X(Y(x, y), \overline{Y(x, y)})}), \overline{Y(Y(x, X(x, y)), X(Y(x, y), \overline{Y(x, y)}))}), \\
& Y(X(y, \overline{Y(x, \bar{x})}), X(X(y, z), \overline{Y(x, \bar{x})}))=X(X(y, z), \overline{Y(x, \bar{x})}), \\
& X(Y(\bar{x}, \overline{X(\bar{x}, x)}), \overline{Y(\bar{x}, X(\bar{x}, x))})=\overline{X(Y(x, \overline{X(x, \bar{x})}), \overline{Y(x, X(x, \bar{x}))})}, \\
& \overline{X(Y(z, \overline{X(z, \bar{z})}), \overline{Y(z, X(z, \bar{z}))})} \\
& =X(\overline{X(Y(u, \overline{X(u, \bar{u})}), \overline{Y(u, X(u, \bar{u}))})}, \overline{X(Y(v, \overline{X(v, \bar{v})}), \overline{Y(v, X(v, \bar{v}))})}) \text {, }
\end{aligned}
$$

где через $z, u$ и $v$ соответственно обозначены термы $X(x, y), X(x, Y(x, y))$ и $X(y, Y(y, x))$,

$$
\begin{aligned}
Y(\overline{X(Y(x, \overline{X(x, \bar{x})}), \overline{Y(x, X(x, \bar{x}))})}, X(x, \bar{x})) & =Y(x, X(x, \bar{x})), \\
Y(\overline{Y(y, X(x, \bar{x}))}, X(x, \overline{Y(x, \bar{x})})) & =x
\end{aligned}
$$


где через $y$ обозначен терм $X(Y(x, \overline{X(x, \bar{x}})), \overline{Y(x, X(x, \bar{x}))})$.

Непосредственной проверкой убеждаемся, что каждое из сверхтождеств (4.9)-(4.27) вьполняется в любой булевой алгебре. Следовательно, согласно определению булевой квазирешетки каждое из них выполняется и в любой булевой квазирешетке.

На множестве $Q$ булевой квазирешетки $\mathfrak{A}=Q\left(+, \cdot,^{-}\right)$определим отношение $\Theta$ следующим образом:

$$
x \Theta y \Longleftrightarrow x+x y=x, \quad y+y x=y .
$$

Из доказательства леммы 3.1 видно, что $\Theta$ - отношение эквивалентности. Пусть $Q=\bigcup_{i \in I} Q_{i}$ - соответствующее разбиение. Каждое подмножество $Q_{i}$ замкнуто относительно сложения и умножения:

$$
\begin{aligned}
a, b \in Q_{i} & \Longrightarrow a \Theta b \Longrightarrow a+a b=a, \quad b+b a=b \\
& \Longrightarrow\left\{\begin{array}{l}
a+a(a+b)=a+a+a b=a+a b+a, \\
a+b+(a+b) a=a+b+a+a b=a+b
\end{array}\right. \\
& \Longrightarrow a \Theta a+b \Longrightarrow a+b \in Q_{i} .
\end{aligned}
$$

Аналогично,

$$
a, b \in Q_{i} \Longrightarrow a \cdot b \in Q_{i} .
$$

Определим новую унарную операцию $x^{\prime}$ на множестве $Q$ следующим образом:

$$
x^{\prime}=x \cdot \overline{x+\bar{x}}+\overline{x \cdot(x+\bar{x})}
$$

и покажем, что каждое подмножество $Q_{i}$ замкнуто и относительно этой унарной операции, т.е. если $a \in Q_{i}$, то $a^{\prime} \in Q_{i}$. Воспользуемся сверхтождествами (4.9), (4.10). Заменяя $X \rightarrow(+), Y \rightarrow(\cdot)$, из сверхтождеств (4.9), (4.10) получим тождества $x+x \cdot x^{\prime}=x, x^{\prime}+x^{\prime} \cdot x=x^{\prime}$. Следовательно, $x \Theta x^{\prime}$ для любого $x \in Q$.

Далее проверяются аксиомы булевых алгебр для каждого $Q_{i}\left(+,{ }^{\prime},\right), \quad i \in I$. Идемпотентность, коммутативность, ассоциативность и дистрибутивность бинарных операций, очевидно, выполняются. Условие $\left(a^{\prime}\right)^{\prime}=a$ вытекает из сверхтождества (4.11) при замене $X \rightarrow(+), Y \rightarrow(\cdot)$. Условие $(a+b)^{\prime}=a^{\prime} \cdot b^{\prime}$ вытекает из сверхтождества (4.12), а условие $a+b \cdot b^{\prime}=a$ для любых $a, b \in Q_{i}$ вытекает из сверхтождества (4.13) при замене $X \rightarrow(+), Y \rightarrow(\cdot)$. Заметим, что нулевой и единичный элементы для $Q_{i}\left(+, \cdot{ }^{\prime}\right)$ будут соответственно:

$$
0_{i}=a \cdot \overline{x+\bar{x}}, \quad 1_{i}=a+\overline{x \cdot \bar{x}}
$$

где $a \in Q_{i}, \quad x \in Q$. Корректность этих определений следует из сверхтождеств (4.14)-(4.17).

Определим операции на фактормножестве $Q / \Theta=\left\{Q_{i} \mid i \in I\right\}$, относительно которых оно также преврашается в булеву алгебру:

$$
Q_{i}+Q_{j}=Q_{k} \Longleftrightarrow 1_{i}+1_{j} \in Q_{k},
$$


где $i, j, k \in I$, при этом $1_{i}+1_{j}=1_{k}$; действительно, если $1_{i}+1_{j} \in Q_{k}$, то

$$
1_{i}+1_{j}=1_{i}+\overline{x \cdot \bar{x}}+1_{j}+\overline{x \cdot \bar{x}}=1_{i}+1_{j}+\overline{x \cdot \bar{x}}=1_{k} ;
$$

б)

$$
\bar{Q}_{i}=Q_{j} \Longleftrightarrow \overline{1}_{i} \in Q_{j}
$$

где $i, j \in I$, при этом $\overline{1}_{i}=0_{j}$; действительно, если $\overline{1}_{i} \in Q_{j}$, то согласно сверхтождеству (4.18) имеем

B)

$$
\overline{1}_{i}=\overline{1_{i}+\overline{x \cdot \bar{x}}}=\overline{1}_{i} \cdot \overline{x+\bar{x}}=0_{j}
$$

$$
Q_{i} \cdot Q_{j}=\overline{\bar{Q}}_{i}+\bar{Q}_{j} .
$$

Из сверхтождеств (4.19), (4.20) вытекают включения $a+b \in Q_{i}+Q_{j}$ и $a \cdot b \in$ $Q_{i}+Q_{j}$, где $a \in Q_{i}, b \in Q_{j}$. Из сверхтождества (4.8) следует включение $\bar{a} \in \bar{Q}_{i}$, где $a \in Q_{i}$.

Проверим аксиомы булевой алгебры для $Q / \Theta\left(+, \cdot,^{-}\right)$:

$\mathrm{B}_{1} \cdot 1_{i}+1_{i}=1_{i} \Longrightarrow Q_{i}+Q_{i}=Q_{i}$;

$\mathrm{B}_{2} \cdot 1_{i}+1_{j}=1_{j}+1_{i} \Longrightarrow Q_{i}+Q_{j}=Q_{j}+Q_{i}$;

$\mathrm{B}_{3} .\left(1_{i}+1_{j}\right)+1_{k}=1_{i}+\left(1_{j}+1_{k}\right) \Longrightarrow\left(Q_{i}+Q_{j}\right)+Q_{k}=Q_{i}+\left(Q_{j}+Q_{k}\right)$;

$\mathrm{B}_{4} \cdot Q_{i} \cdot\left(Q_{j}+Q_{k}\right)=\overline{\bar{Q}_{i}+\overline{Q_{j}+Q_{k}}}$,

$$
\left(Q_{i} \cdot Q_{j}\right)+\left(Q_{i} \cdot Q_{k}\right)={\overline{Q_{i}}+\bar{Q}_{j}}_{1}{\overline{Q_{i}}}+\bar{Q}_{k} .
$$

Из сверхтождества (4.21) имеем

$$
\overline{\overline{1}_{i}+\overline{1_{j}+1_{k}}}=\overline{\overline{1}_{i}+\overline{1}_{j}}+\overline{\overline{1}_{i}+\overline{1}_{k}}
$$

поэтому

$$
Q_{i}\left(Q_{j}+Q_{k}\right)=Q_{i} Q_{j}+Q_{i} Q_{k}
$$

$\mathrm{B}_{5} . \quad \overline{\overline{1}}_{i}=1_{i} \Longrightarrow \overline{\bar{Q}}_{i}=Q_{i}$;

$\mathrm{B}_{6} . \quad \overline{Q_{i} \cdot Q_{j}}=\overline{\overline{\bar{Q}}_{i}+\bar{Q}_{j}}=\bar{Q}_{i}+\bar{Q}_{j}$;

$\mathrm{B}_{7} . \quad a+\overline{b+\bar{b}}=a \Longrightarrow Q_{i}=Q_{i}+\overline{Q_{j}+\overline{Q_{j}}}=Q_{i}+Q_{j} \bar{Q}_{j}$.

Отождествляя каждое $Q_{i}$ со своим индексом, получим булеву алгебру $I(\leqslant)$, где $i \leqslant j \Longleftrightarrow Q_{i} \leqslant Q_{j}$. Пусть $i \leqslant j$. Определим $\varphi_{i, j}: Q_{i} \rightarrow Q_{j}$ по правилу

$$
\varphi_{i, j}(x)=x \cdot 1_{j} \in Q_{i}+Q_{j}=Q_{j}
$$


где $x \in Q_{i}, 1_{j} \in Q_{j}$. Отображение $\varphi_{i, j}$ - гомоморфизм булевых алгебр:

$$
\begin{aligned}
\varphi_{i, j}(x+y) & =(x+y) \cdot 1_{j}=\left(x \cdot 1_{j}\right)+\left(y \cdot 1_{j}\right)=\varphi_{i, j}(x)+\varphi_{i, j}(y), \\
\varphi_{i, j}(x \cdot y) & =(x \cdot y) \cdot 1_{j}=\left(x \cdot 1_{j}\right) \cdot\left(y \cdot 1_{j}\right)=\varphi_{i, j}(x) \cdot \varphi_{i, j}(y) .
\end{aligned}
$$

Для проверки равенства

$$
\varphi_{i, j}\left(x^{\prime}\right)=\left(\varphi_{i, j}(x)\right)^{\prime}
$$

воспользуемся сверхтождеством (4.29), откуда при замене $X \rightarrow(+), Y \rightarrow(\cdot)$ получим

$$
x^{\prime}\left(x y+(x y)^{\prime}\right)=x y \cdot \overline{x y+\overline{x y}}+\overline{x(x+y)(x y+\overline{x y})} .
$$

Следовательно,

$$
\begin{aligned}
\varphi_{i, j}\left(x^{\prime}\right) & =x^{\prime} \cdot 1_{j}=x^{\prime}\left(\left(x \cdot 1_{j}\right)+\left(x \cdot 1_{j}\right)^{\prime}\right) \\
& =x \cdot 1_{j} \cdot \overline{\left(\overline{\left(x \cdot 1_{j}\right)+\overline{x \cdot 1_{j}}}\right)}+\overline{x\left(x+1_{j}\right)\left(\left(x \cdot 1_{j}\right)+\overline{x \cdot 1_{j}}\right)} \\
& =x \cdot 1_{j} \cdot \overline{\left(\left(x \cdot 1_{j}\right)+\overline{x \cdot 1_{j}}\right)}+\overline{x \cdot 1_{j} \cdot\left(\left(x \cdot 1_{j}\right)+\overline{x \cdot 1_{j}}\right)} \\
& =\left(x \cdot 1_{j}\right)^{\prime}=\left(\varphi_{i, j}(x)\right)^{\prime} .
\end{aligned}
$$

Если $i \leqslant j \leqslant k$, то, в частности, $Q_{j}+Q_{k}=Q_{k}$, т.е. $1_{j}+1_{k}=1_{k}$. Проверим равенство $\varphi_{i, j} \cdot \varphi_{j, k}=\varphi_{i, k}$ :

$$
\begin{aligned}
& \left(\varphi_{i, j} \cdot \varphi_{j, k}\right) x=\varphi_{j, k}\left(\varphi_{i, j}(x)\right)=\varphi_{j, k}\left(x \cdot 1_{j}\right) \\
& =x \cdot 1_{j} \cdot 1_{k}=x \cdot 1_{k}=\varphi_{i, k}(x),
\end{aligned}
$$

где $x \in Q_{i}$. Здесь мы воспользовались сверхтождеством (4.23), из которого при $X=(+), Y=(\cdot)$ и $y=1_{j}, z=1_{k}$ следует

$$
\begin{aligned}
1_{j} \cdot 1_{k} & =\left(1_{j}+\overline{x \bar{x}}\right)\left(1_{k}+\overline{x \bar{x}}\right)=\left(1_{j}+\overline{x \bar{x}}\right)\left(1_{j}+1_{k}+\overline{x \bar{x}}\right) \\
& =1_{j}+1_{k}+\overline{x \bar{x}}=1_{k}+\overline{x \bar{x}}=1_{k} .
\end{aligned}
$$

Для любого $i \in I$ определим отображение $h_{i, \bar{i}}: Q_{i} \rightarrow Q_{\bar{i}}$ по правилу

$$
h_{i, \bar{i}}(x)=(\bar{x})^{\prime}=\overline{\left(x^{\prime}\right)} \text {. }
$$

Корректность этого определения вытекает из сверхтождества (4.24) алгебры $Q\left(+, \cdot{ }^{-}\right)$.

Проверим гомоморфность $h_{i, \bar{i}}$, исходя из сверхтождества (4.25) алгебры $Q\left(+, \cdot,-{ }^{-}\right)$. Откуда при замене $X \rightarrow(+)$ и $Y \rightarrow(\cdot)$ получим тождество

$$
\overline{(x+y)^{\prime}}=\overline{(x+x y)^{\prime}}+\overline{(y+y x)^{\prime}} \text {. }
$$

Следовательно,

$$
\begin{aligned}
h_{i, \bar{i}}(x+y) & =\overline{(x+y)^{\prime}}=\overline{(x+x y)^{\prime}}+\overline{(y+y x)^{\prime}} \\
& =\overline{x^{\prime}}+\overline{y^{\prime}}=h_{i, \bar{i}}(x)+h_{i, \bar{i}}(y),
\end{aligned}
$$


где $x, y \in Q_{i}$. Аналогично, с учетом тождества

$$
\overline{(x \cdot y)^{\prime}}=\overline{(x(x+y))^{\prime}} \cdot \overline{(y(y+x))^{\prime}}
$$

получаем

$$
h_{i, \bar{i}}(x \cdot y)=h_{i, \bar{i}}(x) \cdot h_{i, \bar{i}}(y) .
$$

Далее,

$$
h_{i, \bar{i}}\left(x^{\prime}\right)=\left(\overline{x^{\prime}}\right)^{\prime}=\left(h_{i, \bar{i}}(x)\right)^{\prime} .
$$

Из очевидного равенства

$$
h_{i, \bar{i}} \cdot h_{\bar{i}, i}=h_{\bar{i}, i} \cdot h_{i, \bar{i}}=\varepsilon
$$

вытекает биективность отображения $h_{i, \bar{i}}$.

Итак, $h_{i, \bar{i}}-$ изоморфизм булевых алгебр для любого $i \in I$,

$$
\begin{aligned}
\left(h_{i, \bar{i}} \cdot \varphi_{\bar{i}, 1}\right) x & =\varphi_{\bar{i}, 1}\left(h_{i, \bar{i}}(x)\right)=\varphi_{\bar{i}, 1}\left(\overline{x^{\prime}}\right)=\overline{x^{\prime}} \cdot 1_{1} \\
& =\overline{x^{\prime}}(x+\bar{x})=x(x+\bar{x})=x \cdot 1_{1}=\varphi_{i, 1}(x) .
\end{aligned}
$$

Здесь мы воспользовались тождеством

$$
\overline{x^{\prime}}(x+\bar{x})=x(x+\bar{x})
$$

которое возникает из сверхтождества $(4.26)$ алгебры $Q(+, \cdot,-)$.

Докажем биективность $\varphi_{i, j}$. Пусть $i \leqslant j$, и пусть $a_{1}, a_{2} \in Q_{i}$; тогда

$$
\begin{aligned}
\varphi_{i, j}\left(a_{1}\right) & =\varphi_{i, j}\left(a_{2}\right) \Longrightarrow \varphi_{i, 1}\left(a_{1}\right)=\varphi_{j, 1}\left(\varphi_{i, j}\left(a_{1}\right)\right) \\
& =\varphi_{j, 1}\left(\varphi_{i, j}\left(a_{2}\right)\right)=\varphi_{i, 1}\left(a_{2}\right) \Longrightarrow a_{1} \cdot 1_{1}=a_{2} \cdot 1_{1} \\
& \Longrightarrow a_{1}\left(a_{1}+\bar{a}_{1}\right)=a_{2}\left(a_{2}+\bar{a}_{2}\right) \Longrightarrow a_{1}^{\prime} \\
& =a_{1} \cdot \overline{a_{1}+\bar{a}_{1}}+\overline{a_{1}\left(a_{1}+\bar{a}_{1}\right)}=0_{i}+\overline{a_{1}\left(a_{1}+\bar{a}_{1}\right)} \\
& =0_{i}+\overline{a_{2}\left(a_{2}+\bar{a}_{2}\right)}=a_{2} \cdot \overline{a_{2}+\bar{a}_{2}}+\overline{a_{2}\left(a_{2}+\bar{a}_{2}\right)} \\
& =a_{2}^{\prime} \Longrightarrow a_{1}=a_{2} .
\end{aligned}
$$

Для любого $b \in Q_{j}$ обозначим $a=b^{\prime} \cdot 1_{1} \cdot 1_{i} \in Q_{i}$. Тогда

$$
h_{i, j}(a)=\overline{b^{\prime} \cdot 1_{1}} \cdot 1_{i} \cdot 1_{j}=\overline{b^{\prime} \cdot 1_{1}} \cdot 1_{j}=\overline{b^{\prime}(b+\bar{b})} \cdot(b+\overline{b \bar{b}})=b
$$

согласно сверхтождеству (4.27).

Пусть наконец $x, y \in Q=\bigcup_{i \in I} Q_{i}$. Если $x \in Q_{i}, y \in Q_{j}$ и $Q_{i}+Q_{j}=Q_{k}$, т.е. $i+j=k$, то

$$
\begin{gathered}
x+y=(x+y) \cdot 1_{k}=\left(x \cdot 1_{k}\right)+\left(y \cdot 1_{k}\right)=\varphi_{i, k}(x)+\varphi_{j, k}(y), \\
x \cdot y=x \cdot y \cdot 1_{k}=x \cdot 1_{k} \cdot y \cdot 1_{k}=\varphi_{i, k}(x) \cdot \varphi_{j, k}(y), \\
\bar{x}=\overline{\left(x^{\prime}\right)^{\prime}}=\varphi_{i, \bar{i}}\left(x^{\prime}\right) .
\end{gathered}
$$


Таким образом, булева квазирешетка $Q\left(+, \cdot,^{-}\right)$является булевой суммой булевых алгебр $Q_{i}, i \in I$. Теорема 4.2 доказана.

СлЕДСТВИЕ 4.3. Алгебра $\mathfrak{A}=Q\left(+, \cdot,{ }^{-}\right)$с двумя бинарными и одной унарной операциями удовлетворяет регулярным тождествам булевых алгебр тогда и только тогда, когда $\mathfrak{A}$ является булевой алгеброй или булевой суммой булевых алгебр.

Функциональный ранг каждого из сверхтождеств (3.1)-(3.4), (4.1)-(4.7) в силу теоремы 4.1 не больше 3 (считая и единственный унарный символ операции), а предметньй ранг каждого из них не больше 4 . В качестве следствия здесь укажем сушествование такого конечного базиса сверхтождеств многообразия булевых алгебр, для сверхтождеств которого функциональные и предметные ранги не больше 3.

ТЕОРема 4.3. Любое сверхтождество многообразия булевых алгебр является следствием сверхтождеств (3.1)-(3.4), (4.1)-(4.4), (4.8), (4.9)-(4.18), (4.20)-(4.27).

ДокаЗАТЕЛЬСТво. Требуется доказать, что если в $T$-алгебре $\mathfrak{A}=\langle Q ; \Sigma\rangle$, где $T=\{1,2\}$, выполняются указанные в теореме сверхтождества, то в $\mathfrak{A}$ выполняется и любое сверхтождество многообразия булевых алгебр, т.е. $\mathfrak{A}$ является булевой квазирешеткой.

Во-первых, заметим, что если такая алгебра $\mathfrak{A}=\langle Q ; \Sigma\rangle$ подпрямо-неразложима в категории $T$-алгебр, то мощность множества ее бинарных операций $\leqslant 2$. Действительно, при доказательстве леммы 4.1 используются лишь сверхтождества (3.1)-(3.4) и (4.8). Поэтому для рассматриваемой алгебры $\mathfrak{A}$ справедлив и результат типа следствия 4.2 , т.е. в категории $T$-алгебр рассматриваемая алгебра $\mathfrak{A}$ изоморфна подпрямому произведению таких $T$-алгебр $\mathfrak{B}$ со сверхтождествами (3.1)-(3.4), (4.1)-(4.4), (4.8), (4.9)-(4.18), (4.20)-(4.27), множества бинарных операций которых либо одноэлементно, либо двухэлементно. Если алгебра $\mathfrak{B}$ имеет одну бинарную операцию (+), то сверхтождества (3.1)-(3.3), (4.2)-(4.4), (4.21) преврашаются в тождества $\left(4 . \mathrm{B}_{1}\right)-\left(4 . \mathrm{B}_{7}\right)$, которые обеспечивают вложение этой алгебры в булеву алгебру. Поэтому в такой алгебре $\mathfrak{B}$ будет выполняться каждое сверхтождество многообразия булевых алгебр. Если же алгебра $\mathfrak{B}$ имеет две бинарные операции, то она является булевой алгеброй или представима в виде булевой суммы булевых алгебр, поскольку при доказательстве необходимости теоремы 4.2 на самом деле используются лишь сверхтождества (3.1)-(3.4), (4.1)-(4.4), (4.8), (4.9)-(4.18), (4.20)-(4.27) или их следствия (например, сверхтождество (4.19), имеющее предметный ранг, равньй 4, согласно теореме 3.1 является следствием сверхтождеств (3.1)-(3.4)). Далее, согласно достаточности теоремы 4.2 в алгебре $\mathfrak{B}$ будет выполняться любое сверхтождество многообразия булевых алгебр. В результате и в рассматриваемой алгебре $\mathfrak{A}$ будет выполняться любое сверхтождество многообразия булевых алгебр. Теорема 4.3 доказана.

СледСтвИЕ 4.5. Любое сверхтождество булевой алгебры является следствием сверхтождеств (3.1)-(3.4), (4.1)-(4.4), (4.8), (4.9)-(4.18), (4.20)-(4.27), функциональнье и предметные ранги которьх не больше 3. 
СЛЕДСТВИЕ 4.6. Любое регулярное тождество булевой алгебры является следствием конечной системы тождеств, полученных из сверхтождеств (3.1)-(3.4), (4.2)-(4.4), (4.8), (4.9)-(4.18), (4.20)-(4.27) при заменах $X \rightarrow(+),(\cdot)$ u $Y \rightarrow(+),(\cdot)$.

\section{Список литературы}

1. Чёрч А. Введение в математическую логику. Т. 1. М.: ИЛ, 1961.

2. Мальцев А. И. Алгебраические системы. М.: Наука, 1970.

3. Мовсисян Ю. М. Введение в теорию алгебр со сверхтождествами. Ереван: Изд-во ЕГУ, 1986.

4. Мовсисян Ю. М. Сверхтождества и сверхмногообразия в алгебрах. Ереван: Изд-во ЕГУ, 1990.

5. Мальцев А.И. Модельные соответствия // Изв. АН СССР. Сер. матем. 1959. Т. 23. № 3. С. $313-336$.

6. Мальцев А.И. Некоторые вопросы теории классов моделей // Tp. IV Всесоюзного математического съезда. 1963. Т. 1. С. 169-198.

7. Кейслер Г., Чэн Ч. Теория моделей. М.: Мир, 1977.

8. Schauffler R. Die Assoziativität im Ganzen besonders bei Quasigruppen // Math. Zeitschr. 1957. B. 67. № 5. S. 428-435.

9. Denes J., Keedwell A.D. Lattin squares and their applications. Budapest: Academiai Kiado, 1974.

10. Белоусов В. Д. Системы квазигрупп с обобщенными тождествами // УМН. 1965. Т. 20. № 1. С. $75-146$.

11. Мовсисян Ю. М. К теореме Шауфлера // Матем. заметки. 1993. Т. 53. № 2. С. 84-93.

12. Taylor $W$. Hyperidentities and hypervarieties // Aequationes Math. 1981. V. 23. P. 30-49.

13. Мовсисян Ю. М. Сверхтождества булевых алгебр // Изв. АН. Сер. матем. 1992. Т. 56. C. $654-672$.

14. Скорняков Л.А. Стохастическая алгебра // Изв. ВУЗов. Математика. 1985. № 7. C. $3-11$.

15. Romanowska A. B., Smith J. D. H. Modal Theory. Berlin: Heldermann Verlag, 1985.

16. Биркгоф Г. Теория решеток. М.: Наука, 1974.

17. Moufang R. Zur structure von Alternativ Körpern // Math. Ann. 1935. V. 110. P. 416-430.

18. Padmanabhan R., Penner P. Bases of hyperidentities of lattices and semilattices // C. R. Math. Rep. Acad. Sci. Canada. 1982. V. 4. P. 9-14.

19. Bergman G. M. Hyperidentities of groups and semigroups // Aequationes Math. 1981. V. 23. P. 50-65.

20. Denecke K., Pöschel $R$. The characterization of primal algebras by hyperidentities. Preprint, 1988.

21. Denecke K., Pöschel $R$. A characterization of Sheffer functions by hyperidentities // Semigroup Forum. 1988. V. 37. P. 351-362.

22. Schweigert D., Denecke K. Hyperidentities and completeness properties of finite algebras. Preprint, 1987.

23. Mal'cev I. A., Schweigert D. Hyperidentitaten von QZ-algebren. Preprint, 1989.

24. Reischer C., Schweigert D., Simovici D.A. A completeness criterion by functional equations. Preprint, 1987.

25. Denecke $K$. Eine Charakterisierung der funktionalen Vollständigkeit in kongruenzvertauschbaren Varietäten durch Hyperidentitäten // Rostock. Math. Kolloq. 1989. V. 36. P. $73-80$.

26. Schweigert D. Clones of term functions of lattices and abelian groups // Algebra Universalis. 1985. V. 20. P. 27-33.

27. Graczynska E. Connections between identities and hyperidentities // Bull. of the Section of Logik (Warsaw-Lodz). 1988. V. 17. P. 34-41. 
28. Penner P. Hyperidentities of semilattices // Houston J. Math. 1984. V. 10. P. 81-108.

29. Aczel J. Proof of a theorem of distributive type hyperidentities // Algebra Universalis. 1971. V. 1. P. 1-6.

30. Marczewski E. A general scheme of the notions of independence in mathematics // Bull. Acad. Polon. Sci. Ser. Math., Astron., Phys. 1958. V. 6. P. 731-736.

31. Гретцер Г. Общая теория решеток. М.: Мир, 1977.

32. Plonka J. On distributive $n$-lattices and $n$-quasilattices // Fundamenta Mathematicae. 1968. V. 62. P. 293-300.

33. Plonka J. On distributive quasi-lattices // Fundamenta Mathematicae. 1967. V. 60. P. 191-200.

34. Padmanabhan R. Regular identities in lattices // Transaction of the American Math. Society. 1971. V. 158. P. 179-188.

35. Plonka J. On a method of construction of abstract algebras // Fundamenta Mathematicae. 1967. V. 61. P. 183-189.

Ереванский государственный университет

Поступило в редакцию 28.VI.1995 